\title{
High-contrast integral field spectropolarimetry of planet-forming disks with SCEXAO/CHARIS
}

Lawson, Kellen, Currie, Thayne, Wisniewski, John, Hashimoto, Jun, Guyon, Olivier, et al.

Kellen Lawson, Thayne Currie, John P. Wisniewski, Jun Hashimoto, Olivier Guyon, N, Jeremy Kasdin, Tyler D. Groff, Julien Lozi, Timothy D. Brandt, Jeffrey Chilcote, Vincent Deo, Taichi Uyama, Sebastien Vievard, "Highcontrast integral field spectropolarimetry of planet-forming disks with SCExAO/CHARIS," Proc. SPIE 11823, Techniques and Instrumentation for Detection of Exoplanets X, 118230D (1 September 2021); doi: 10.1117/12.2594819

Event: SPIE Optical Engineering + Applications, 2021, San Diego, California, United States 


\title{
High-contrast integral field spectropolarimetry of planet-forming disks with SCExAO/CHARIS
}

\author{
Kellen Lawson ${ }^{\mathrm{a}}$, Thayne Currie ${ }^{\mathrm{b}, \mathrm{c}, \mathrm{d}}$, John P. Wisniewski ${ }^{\mathrm{a}}$, Jun Hashimoto ${ }^{\mathrm{e}, \mathrm{b}, \mathrm{f}}$, Olivier \\ Guyon $^{\mathrm{b}, \mathrm{g}, \mathrm{e}}$, N. Jeremy Kasdin ${ }^{\mathrm{h}}$, Tyler D. Groffi, Julien Lozi ${ }^{\mathrm{b}}$, Timothy D. Brandt ${ }^{\mathrm{j}}$, Jeffrey \\ Chilcote $^{\mathrm{k}}$, Vincent Deo ${ }^{\mathrm{b}}$, Taichi Uyama, ${ }^{\mathrm{l}, \mathrm{m}}$, and Sebastien Vievard ${ }^{\mathrm{b}}$ \\ aDepartment of Physics and Astronomy, University of Oklahoma, Norman, OK, USA \\ ${ }^{b}$ Subaru Telescope, National Astronomical Observatory of Japan, 650 North A'ohōk $\bar{u}$ Place, \\ Hilo, HI, USA \\ 'NASA-Ames Research Center, Moffett Blvd., Moffett Field, CA, USA \\ ${ }^{\mathrm{d}}$ Eureka Scientific, 2452 Delmer Street Suite 100, Oakland, CA, USA \\ eAstrobiology Center, National Institutes of Natural Sciences, 2-21-1, Osawa, Mitaka, Tokyo, \\ 181-8588, Japan \\ ${ }^{\mathrm{f}}$ Department of Astronomy, School of Science, Graduate University for Advanced Studies \\ (SOKENDAI), Mitaka, Tokyo 181-8588, Japan \\ ${ }^{\mathrm{g}}$ College of Optical Sciences, University of Arizona, Tucson, AZ, USA \\ ${ }^{\mathrm{h}}$ Department of Mechanical Engineering, Princeton University, Princeton, NJ, USA \\ iNASA-Goddard Space Flight Center, Greenbelt, MD, USA \\ jDepartment of Physics, University of California, Santa Barbara, Santa Barbara, California, \\ USA \\ ${ }^{\mathrm{k}}$ Department of Physics, University of Notre Dame, South Bend, IN, USA \\ ${ }^{1}$ Infrared Processing and Analysis Center, California Institute of Technology, 1200 E. \\ California Boulevard, Pasadena, CA 91125, USA \\ mASA Exoplanet Science Institute, Pasadena, CA 91125, USA
}

\begin{abstract}
We describe a new high-contrast imaging capability well suited for studying planet-forming disks: near-infrared (NIR) high-contrast spectropolarimetric imaging with the Subaru Coronagraphic Extreme Adaptive Optics (SCExAO) system coupled with the Coronagraphic High Angular Resolution Imaging Spectrograph (CHARIS) integral field spectrograph (IFS). The advent of extreme adaptive optics (AO) systems, like SCExAO, has enabled recovery of planet-mass companions at the expected locations of gas-giant formation in young disks alongside disk structures (such as gaps or spirals) that may indicate protoplanet formation. In combination with SCExAO, the CHARIS IFS in polarimetry mode allows characterization of these systems at wavelengths spanning the NIR J, H, and $\mathrm{K}$ bands $(1.1-2.4 \mu \mathrm{m}, R \sim 20)$ and at angular separations as small as $0{ }^{\prime \prime} 04$. By comparing the resulting images with forward-modeled scattered light or $3 \mathrm{D}$ radiative-transfer models, the likely origins of any observed features can be assessed. Utilization of swift optimization algorithms, such as differential evolution (DE), to identify model parameters that best reproduce the observations allows plausible disk geometries to be explored efficiently. The recent addition of CHARIS's unique integral field spectropolarimetry mode has further facilitated the study of planet-forming disks - aiding in the confirmation of candidate protoplanets, the diagnosis of disk structures, and the characterization of dust grain populations. We summarize preliminary results for two young planet-forming disk systems based on observations with the novel integral field spectropolarimetry mode for SCExAO/CHARIS.
\end{abstract}

Keywords: Polarimetry, circumstellar disks, exoplanets, high-contrast imaging, extreme adaptive optics

Further author information: (Send correspondence to K. Lawson)

K. Lawson: E-mail: kellenlawson@gmail.com

Techniques and Instrumentation for Detection of Exoplanets X, edited by Stuart B. Shaklan, Garreth J. Ruane, Proc. of SPIE Vol. 11823, 118230D · @ 2021 SPIE · CCC code: 0277-786X/21/\$21 · doi: 10.1117/12.2594819 


\section{INTRODUCTION}

Protoplanetary disks serve as benchmark systems to study how and where exoplanets form. With the advent of ground-based extreme adaptive optics (AO) facilities, such as Spectro-Polarimetric High-contrast Exoplanet REsearch instrument (SPHERE), ${ }^{1}$ Gemini Planet Imager (GPI), ${ }^{2}$ and Subaru Coronagraphic Extreme Adaptive Optics (SCExAO), ${ }^{3-5}$ we have entered a revolutionary era in the study of young protoplanetary disks. It is now feasible to both a) spatially resolve the morphological signatures within disks that could be caused by newly formed/forming sub-stellar objects and planets (such as gaps or spirals), and b) identify young candidate planets and sub-stellar objects that may cause them (e.g. Refs 6,7).

Differential imaging techniques for high-contrast imaging in total intensity, such as angular differential imaging (ADI), ${ }^{8}$ spectral differential imaging (SDI), ${ }^{9}$ and reference star differential imaging (RDI), attempt to disentangle the light of the bright stellar point spread function (PSF) from that of any circumstellar sources by modeling and subtracting the starlight in a data sequence. While these techniques are generally reasonably well suited for the recovery and characterization of isolated point-like companion candidates, the presence of signal from circumstellar disks can significantly inhibit their efficacy - resulting in products in which disk signal is substantially attenuated. For ADI and SDI, this results from self-subtraction (where disk signal is erroneously included in the model PSF) and oversubtraction (where the presence of disk signal in the data results in a brighter PSF model than if it were not present). ${ }^{10,11}$ While RDI is expected to perform better for disk imaging - by eliminating the possibility of self-subtraction - oversubtraction still occurs, and can be significant for bright disks or disks which dominate the field of view (FOV). As a result, any analysis of disks recovered from total intensity differential imaging will generally require the attenuation to be quantified. Further, rigorous assessment of planet candidates embedded in disk material requires a means by which to differentiate a planet's signal from that of disk substructure (e.g. Refs 12,13). Forward-modeling of synthetic disk models is a common approach for approximating disk attenuation (e.g. Refs 13,14). Combined with integral field spectroscopy or multi-wavelength imaging, this can also provide a means by which to validate or invalidate candidate embedded planets. However, given the complexity of total intensity PSF-subtraction and of the geometry of many protoplanetary disks, forward-modeling can be prohibitively time-consuming.

High-contrast near-infrared (NIR) polarimetric imagers - such as Gemini South's GPI, ${ }^{2,15}$ VLT's SPHEREInfraRed Dual-band Imager and Spectrograph (IRDIS), ${ }^{16,17}$ and Subaru's High-Contrast Coronographic Imager for Adaptive Optics (HiCIAO) $)^{18}$ - have been prolific in detecting and characterizing circumstellar disks through the use of another differential imaging technique: polarimetric differential imaging (PDI). While PDI is limited to the recovery of polarized flux, it provides an important complement for total intensity differential imaging by producing a nearly unattenuated view of the disk. Additionally, since self-luminous sub-stellar objects and exoplanets are expected to be unpolarized while light from spatially resolved circumstellar disks is $25-50 \%$ polarized, ${ }^{19,20}$ comparison of total and polarized intensity imaging can serve as another powerful tool for disentangling planet signals from disk sub-structure. Since the PDI procedure is computationally simple, forward modeling disks to simulate the effects of PDI is substantially less time consuming - thus allowing for more detailed investigation of disk model parameters.

Since the decommissioning of Subaru's HiCIAO, the Coronagraphic High Angular Resolution Imaging Spectrograph (CHARIS) NIR integral field spectrograph (IFS) ${ }^{21}$ (paired with SCExAO) has served as Subaru's NIR total intensity high-contrast imager. More recently, CHARIS was upgraded to include spectropolarimetric capabilities as well. ${ }^{22}$ Unprecedented among extreme AO imagers, this new spectropolarimetric imaging mode allows polarimetric observations to be conducted at the same array of wavelengths as its classical (total intensity) observing mode. This capability makes accessible many novel investigations relevant for protoplanetary disk studies, such as characterization of the wavelength dependence of the scattering phase function in disks.

We present preliminary science products from the novel integral field spectropolarimetry mode, or PDImode, for Subaru's SCExAO/CHARIS. Combining the benefits of multi-wavelength IFS imaging with those of polarimetric imaging, this new observing mode provides an exciting tool for groups studying circumstellar disks. Herein, we: 1) provide a summary of the CHARIS PDI-mode, outline its available configurations, and make recommendations for those observing with this mode (Section 2), 2) provide a description of the data processing and calibration procedures for CHARIS PDI data, implemented in a new module for the CHARIS Data Processing Pipeline (DPP) (Section 3), 3) present preliminary science products for two protoplanetary disk 
targets observed with CHARIS in PDI-mode (Section 4), 4) demonstrate disk forward-modeling for CHARIS PDI data (Section 5), and 5) conclude by summarizing compelling future use-cases for CHARIS's PDI-mode in application to the study of circumstellar disks (Section 6).

\section{CHARIS SPECTROPOLARIMETRY MODE}

The spectropolarimetric observing mode for CHARIS is enabled by the addition of a field stop and Wollaston prism upstream of the imager in combination with the existing half-wave plate (HWP) - originally added to enable polarimetry with HiCIAO. The Wollaston prism serves to split incoming light to two orthogonally polarized states, while the HWP enables shifting of the polarization direction being measured, and the field stop eliminates cross-talk between the two polarizations*. Together, the Wollaston prism and HWP enable measurement of linear polarization parameters (Stokes- $Q$ and $U$; in addition to the total intensity, Stokes- $I$, measured by the classical-mode), at CHARIS's typical array of wavelengths, at the cost of reducing CHARIS's FOV from $\sim 2^{\prime \prime} \times 2^{\prime \prime}$ to $\sim 1^{\prime \prime} \times 2^{\prime \prime}$. In the typical PDI procedure, circumstellar polarized flux is isolated by making observations at HWP angles of $0^{\circ}$ and $45^{\circ}$ or $22^{\circ} .5$ and 67.5 and using "double-differencing" (see a full description of PDI in Section 3.4).

A complete model for the correction of instrumental polarization for CHARIS PDI-mode is near completion (see Ref 23) and will be implemented in the data processing pipeline for CHARIS soon after. In lieu of this correction, results herein should be considered preliminary.

\subsection{Half-wave Plate Cycles}

Switching of the HWP requires $\sim 6$ seconds. While the most straight-forward strategy might be to change the HWP angle between each exposure, this could introduce significant overheads for targets requiring short exposure times. Especially in these cases, taking multiple exposures at a given HWP position before each switch may improve the quality of results by enabling more time on target overall. This introduces two possible costs, however. The first is that the suppression of unpolarized stellar flux may be compromised by evolution of the diffraction pattern over the relatively protracted HWP cycles. Additionally, since SCExAO operates exclusively in pupil-tracking mode, exposures at paired HWP positions for double differencing will be at slightly different parallactic angles. This could result in noticeable smearing of circumstellar features for targets observed near zenith (though the impact of this is unlikely to be significant, see: Section 5). As such, target properties, observing conditions, and the circumstellar features of interest should all be considered when planning observations.

\subsection{Instrument Configurations}

With some exceptions, the available configurations for CHARIS's PDI-mode are the same as for its classical-mode. These configurations are summarized hereafter.

Astrogrid - As with CHARIS's classical imaging mode, CHARIS PDI-mode allows for use of the astrogrid during observations. The astrogrid introduces a set of satellite spots which are attenuated copies of the unocculted stellar PSF. The astrogrid enables high quality image registration and flux calibration even when the star is obscured by a coronagraph. While the same array of astrogrid spacings are available as for CHARIS's classical mode, the standard classical-mode spacing of $15.9 \lambda / D$ places spots outside of the narrower $2^{\prime \prime} \times 1^{\prime \prime}$ FOV of PDI-mode by the latter channels of H-band. Thus a spacing of $\sim 11.2 \lambda / D$ or smaller should be utilized for the PDI-mode. ${ }^{22}$ While a limited sequence of exposures utilizing the astrogrid might be sufficient for reasonable registration and flux calibration, use of the astrogrid throughout the entirety of an observing sequence is strongly recommended unless a compelling science case exists to preclude this - such as simultaneous use of the Visible Aperture Masking Polarimetric Imager for Resolved Exoplanetary Structures (VAMPIRES) instrument, which cannot be used with the astrogrid on.

Filters and Spectral Resolution - J-band $(1.18-1.33 \mu \mathrm{m}), H$-band $(1.49-1.78 \mu \mathrm{m})$, and $K$-band $(2.02-2.38 \mu \mathrm{m})$ are available with high spectral resolution $(R \sim 70-90$, yielding $15-20$ wavelength channels in extracted image cubes). CHARIS broadband $(1.15-2.39 \mu \mathrm{m})$, and Cold ND3 $(1.17-2.37 \mu \mathrm{m})$ are available with low spectral resolution ( $R \sim 19$, yielding 22 wavelength channels in extracted image cubes).

\footnotetext{
*see Refs 22,23 for a more complete description of the instrumental design of the CHARIS spectropolarimetry mode
} 
Coronagraphs - the Lyot coronagraphs ${ }^{\dagger}$ can be used with CHARIS PDI-mode for any available filter. Additionally, the phase-induced amplitude apodization complex mask coronagraph (PIAACMC) can be used when observing in $H$-band. Other coronagraphs that are generally available for SCExAO, e.g. vector Apodizing Phase Plate (vAPP) and vortex coronagraphs, induce intractable changes in the polarization state of incident light and are thus unsuitable for observations in PDI-mode.

\subsection{Calibration Data}

With the complete Mueller matrix model for instrumental polarization implemented, observations of polarized/unpolarized standard stars will not be directly necessary for calibration of science products. However, these observations are still recommended, as they require very little time, can be used to verify the integrity of the instrumental polarization corrections, and can be useful in PSF-subtraction of total-intensity products by enabling RDI.

\subsection{Observations}

To demonstrate the use of the methods described herein and to showcase this new observing mode, we include preliminary results of observations of two protoplanetary disk-bearing stars. In both cases, we reserve detailed analysis, discussion, and target-specific conclusions for future publications.

AB Aurigae- AB Aurigae $\left(1-3 \mathrm{Myr},{ }^{24} d=156 \mathrm{pc}^{25,26}\right)$ is a pre-main sequence star that hosts a complex protoplanetary disk bearing spiral arms at scales from tens of au ${ }^{27,28}$ to hundreds. ${ }^{29,30}$

On 2020 October 4, we observed AB Aur with SCExAO/CHARIS in low-res $(R \sim 19)$, broadband $(1.15-2.39$ $\mu m$ ), PDI-mode, using a 113 mas radius Lyot coronagraph and a four-spot astrogrid of spacing $11.2 \lambda / D$. AO performance was average / below-average, with a strong wind-driven halo. We collected 73 science exposures of 60.48 seconds each for a combined integration time of $\sim 73.6$ minutes, achieving a total field rotation of 79.95. Sky frames were also collected at the end of the sequence.

Additional observations were made earlier in the same night using an astrogrid spacing of $15.9 \lambda / D$. Since the satellite spots are not recoverable in every channel at this spacing, image calibration for this data is much less precise. As such, we present only the observations with $11.2 \lambda / D$ astrogrid spacing herein.

TW Hydrae- TW Hydrae $\left(3-10 \mathrm{Myr},{ }^{31-34} d=60 \mathrm{pc}^{25,26}\right)$ is a nearby T-Tauri star with a nearly face-on disk which shows multiple radial gaps that could suggest the presence of planets or their ongoing formation. ${ }^{34,35}$

On 2021 March 20, we observed TW Hya with CHARIS in the same configuration as for AB Aur, but using a smaller Lyot coronagraph (78 mas radius). The AO performance for TW Hya was worse than that of $\mathrm{AB}$ Aur, largely as a result of TW Hya's fainter apparent magnitude and lower altitude $\left(\sim 35^{\circ}\right.$ versus $\gtrsim 74^{\circ}$ for AB Aur). We collected 52 science exposures of 60.48 seconds each for a combined integration time of $\sim 52.4$ minutes, achieving a total field rotation of 15.84 . No sky frames were collected with these data.

\section{CHARIS PDI DATA REDUCTION AND PROCESSING}

\subsection{Cube Extraction}

CHARIS PDI data were extracted from raw IFS exposures using the CHARIS Data Reduction Pipeline (DRP) ${ }^{36}$ with modifications to read noise suppression provided in Ref 5 . The 22 wavelength channels of a single extracted AB Aur image cube are shown in Figure 1.

\footnotetext{
${ }^{\dagger}$ See https://www.naoj.org/Projects/SCEXAO/ for information regarding available coronagraph sizes.
} 

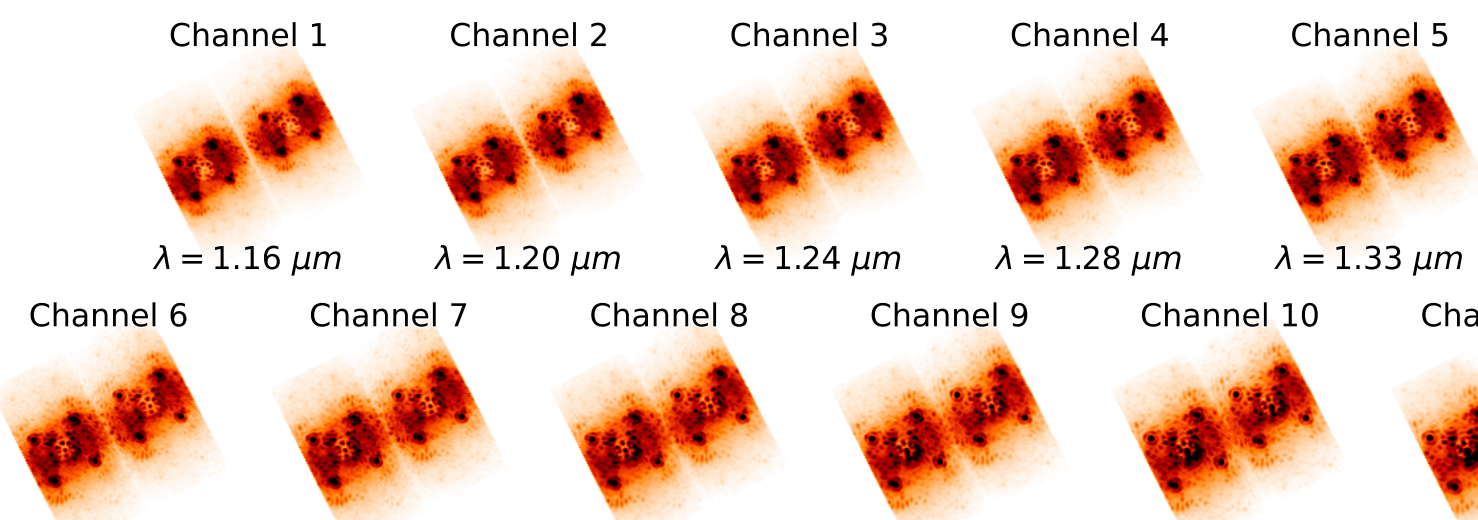

Channel 11

$\lambda=1.37 \mu \mathrm{m}$

$\lambda=1.42 \mu \mathrm{m}$

$\lambda=1.47 \mu \mathrm{m}$

$\lambda=1.52 \mu \mathrm{m}$

$\lambda=1.58 \mu \mathrm{m}$

$\lambda=1.63 \mu \mathrm{m}$

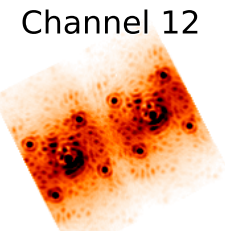

Channel 13
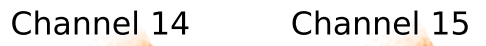

Channel 16

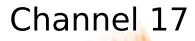

$\lambda=1.69 \mu \mathrm{m}$
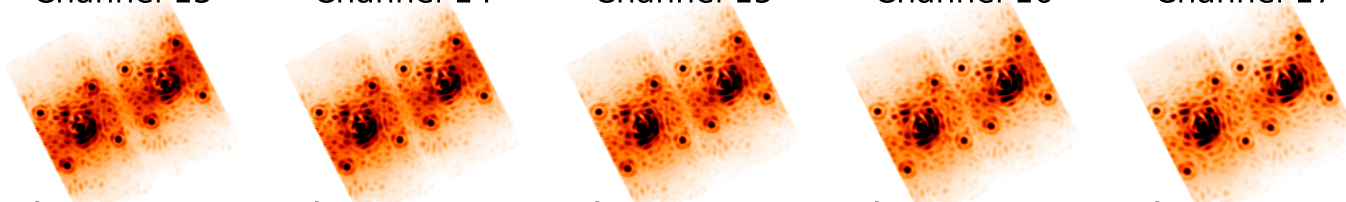

Channel 18

Channel 19

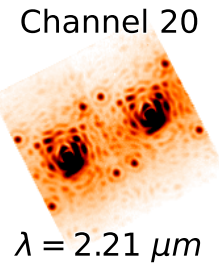

Channel 21

Channel 22
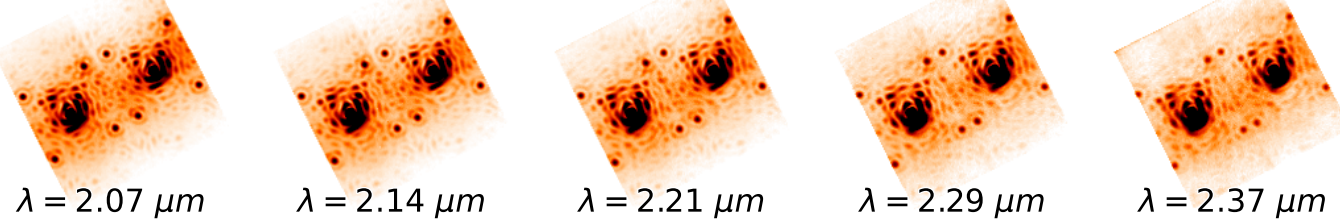

Figure 1. The 22 individual wavelength channels of an extracted low-res, broadband, PDI-mode CHARIS exposure of AB Aur - showing both left and right polarizations. For this exposure, a four-satellite-spot astrogrid with spacing of 11.2 $\lambda / D$, and a Lyot-coronagraph of radius 113 mas were utilized.

\subsection{Preprocessing}

Preliminary processing of data for PDI-mode - e.g. sky subtraction, image registration, and spectrophotometric calibration - is carried out largely as typical for CHARIS classical-mode data, ${ }^{37}$ with the following caveats.

1. Registration - the left and right polarized halves of each image cube are first split into separate files. The sets of left and right frames are then registered independently, but otherwise as standard for CHARIS data (e.g. using satellite spots for occulted data, or PSF centroiding for unocculted data).

2. Spectrophotometric Calibration - for this purpose, the sets of registered left and right polarization cubes are added to form a sequence of single sum total intensity images. These images are then flux calibrated in the same manner as classical-mode data - resulting in a set of scaling factors for each of the 22 wavelength channels. These scaling factors are then applied to the original constituent left and right polarized image cubes.

\subsection{Half-Wave Plate Cycle Matching}

To enable PDI using double-differencing, we group observations into sets containing exposures at each of the four HWP positions. While sets of two images (with HWP angles of $0^{\circ}$ and $45^{\circ}$ or 22.5 and $67^{\circ} .5$ ) would be sufficient to enable double-differencing (see Section 3.4), forming groups of complete HWP cycles allows polarized intensity outputs for each individual cycle - which can be helpful in evaluating the validity of marginally detected features in products for the fully-combined observing sequence. 
Here, each exposure is used only once, and is grouped to minimize parallactic angle differences between the exposures in a cycle. Ultimately, this procedure results in a number of full HWP cycles equal to the minimum number of observations for any one HWP position after low-quality or otherwise problematic exposures are eliminated. Any unmatched exposures are not utilized in the PDI procedure.

\subsection{Polarimetric Differential Imaging}

Reduction of the preprocessed data sequence to final polarimetry products is carried out using the typical double differencing procedure for each HWP cycle (see e.g. Ref 16). First, single sums and differences are computed for each exposure (adopting the notation of Ref 23):

$$
\begin{aligned}
& X^{ \pm}=I_{\text {det }, L}-I_{d e t, R}, \\
& I_{X^{ \pm}}=I_{d e t, L}+I_{d e t, R},
\end{aligned}
$$

where $I_{\text {det }, L}$ and $I_{\text {det }, R}$ are the left and right images respectively, $X^{ \pm}$are the single differences $-Q^{+}, U^{+}, Q^{-}$, $U^{-}-$and $I_{X^{ \pm}}$are the single sums $-I_{Q^{+}}, I_{U^{+}}, I_{Q^{-}}, I_{U^{-}}$- for exposures at HWP angles of $0^{\circ}, 22^{\circ} .5,45^{\circ}$, and 67.5 respectively. From these, double sums and differences are computed as:

$$
\begin{gathered}
X=\frac{1}{2}\left(X^{+}-X^{-}\right), \\
I_{X}=\frac{1}{2}\left(I_{X^{+}}+I_{X^{-}}\right),
\end{gathered}
$$

where the double difference, $X$, corresponds to Stokes $Q$ or $U$, and the double sum, $I_{X}$, corresponds to the components of Stokes $I$ : $I_{Q}$ or $I_{U}$. At this stage, in lieu of a final model for instrumental polarization, we make a first order approximation of instrumental polarization following the procedure of Ref 16 and summarized hereafter. For each double difference image cube, $X$, resulting from the procedure above, the ratio with the corresponding double-sum image cube, $I_{X}$, is computed: $x=X / I_{X}$. Then, in each wavelength channel, $i$, we calculate the median value of $x$ within a circular stellocentric aperture (excluding the inner occulted region if a coronagraph was utilized): $c_{X}^{i}$. The first-order instrumental polarization subtracted cube is then:

$$
X_{\mathrm{IPS}}=X-c_{X} \cdot I_{X}
$$

where $c_{X}$ is the array of coefficients for the $N_{\lambda}$ wavelength channels, $c_{X}=\left[c_{X}^{1}, c_{X}^{2}, \ldots, c_{X}^{N_{\lambda}}\right]$. Since this procedure assumes that the polarization measured in the aperture should be zero in the absence of instrumental polarization, the coverage of the aperture should be chosen to minimize inclusion of circumstellar material. An example of a single wavelength channel of Stokes parameter images resulting from this procedure is shown in Figure 2.

Once $Q_{\text {IPS }}$ and $U_{\text {IPS }}$ (hereafter simply $Q$ and $U$ ) are attained, the products can be derotated to a "north-up" orientation. Noting that a) $X^{+}$and $X^{-}$are not perfectly contemporaneous, and b) SCExAO observes exclusively in pupil-tracking mode: the constituent images will have slightly different angular offsets from north-up. Here, we simply use the average of the parallactic angles of the two constituent frames to compute the rotation needed for a north-up orientation. The effect of this difference in parallactic angle is assessed in Section 5.

From the north-up polarization products, we can compute a few distinct polarized intensity products. Generally:

$$
\mathrm{PI}=\sqrt{\mathrm{Q}^{2}+\mathrm{U}^{2}}
$$

where PI is the polarized intensity. For a single HWP cycle:

1. a corresponding PI cube can be created,

2. a selection of wavelength slices of the $Q$ and $U$ cubes can be combined to approximate common NIR filters (e.g., $J, H$ or $K$ bands), from which a corresponding PI image can be calculated, 

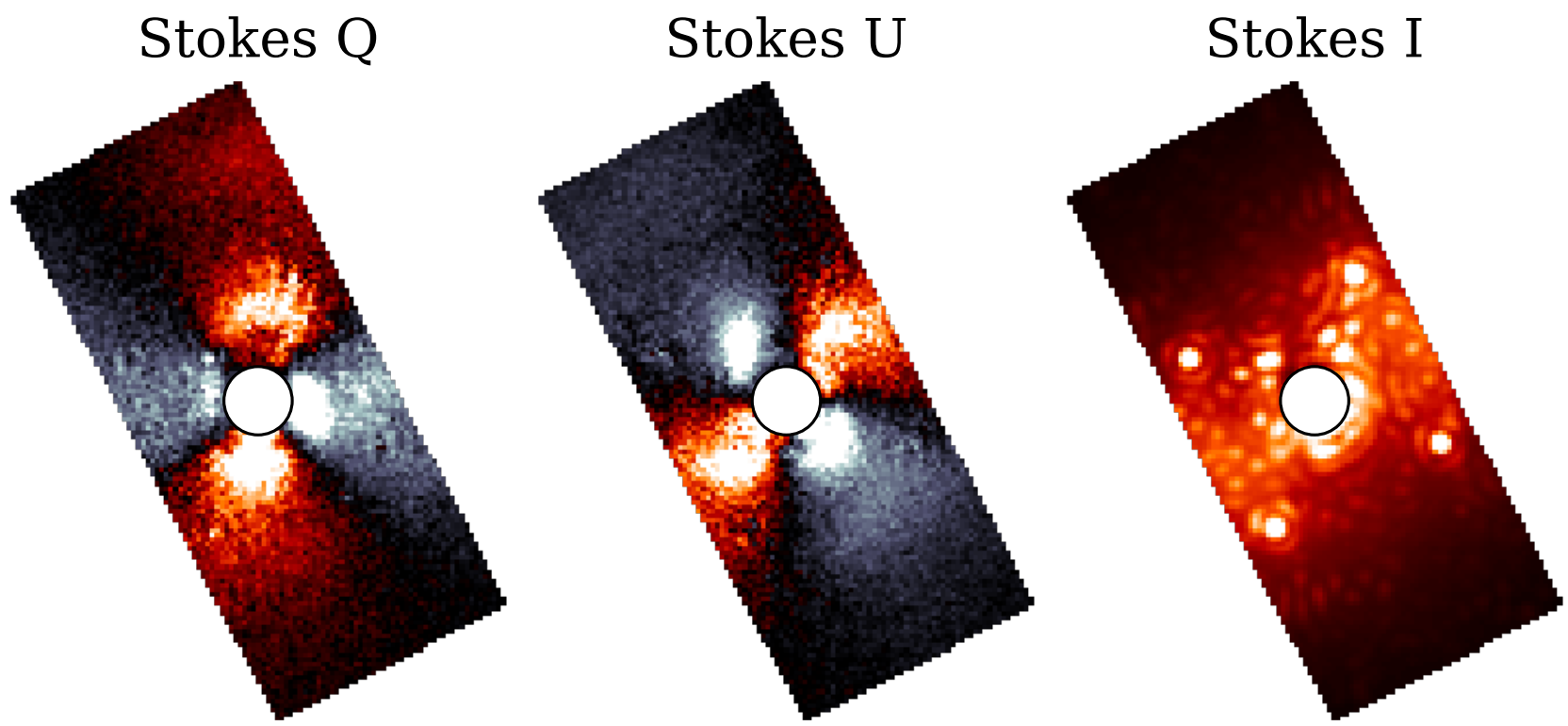

Figure 2. A single wavelength channel (channel $11, \lambda=1.63 \mu \mathrm{m}$ ) showing Stokes $Q, U$, and $I$ images resulting for one HWP cycle of CHARIS AB Aur data (including the exposure of Figure 1). $I$ is computed from the $I_{Q}$ and $I_{U}$ products for the cycle as $I=0.5\left(I_{Q}+I_{U}\right)$. $Q$ and $U$ images have a first order instrumental polarization correction applied. Color stretches are symmetric about zero, with the range for $I$ differing from that of $Q$ and $U$ for visualization. The frames have not yet been derotated.

3. all wavelength slices for $Q$ and $U$ cubes can be combined to enable calculation of a higher signal-to-noise ratio (SNR) PI image.

Additionally, we can average the full set of $Q$ and $U$ image cubes over all cycles to produce a sequencecombined $Q$ and $U$ image cube. From this, the same array of options for presenting PI are available. Since the nominal $1^{\prime \prime} \times 2^{\prime \prime}$ FOV has been derotated by varying amounts, a sequence-combined product will have full coverage over $r \lesssim 0^{\prime \prime} 5$ and partial coverage of a larger area - approaching a $\sim 1^{\prime \prime}$ circle of partial coverage when a total field rotation of $\Delta \mathrm{PA} \sim 125^{\circ}$ is achieved. For $\Delta \mathrm{PA} \lesssim 125^{\circ}$, the region of zero coverage in a sequencecombined product manifests as two opposing wedges extending from $r \gtrsim 0^{\prime \prime} 5$ (see Figure 3 ). We note, however, that the exact FOV recovered will depend on the details of the dataset.

For each of these cases, we can also calculate the angle of linear polarization (AOLP):

$$
\mathrm{AOLP}=\frac{1}{2} \arctan \left(\frac{\mathrm{U}}{\mathrm{Q}}\right)
$$

as well as the azimuthal Stokes parameters:

$$
\begin{gathered}
Q_{\phi}=-Q \cos (2 \phi)-U \sin (2 \phi), \\
U_{\phi}=Q \sin (2 \phi)-U \cos (2 \phi),
\end{gathered}
$$

where $\phi$ is the pixel-wise azimuthal angle relative to the image center.

\section{PRELIMINARY CHARIS SPECTROPOLARIMETRY RESULTS}

We apply the methods of Section 3 to the CHARIS PDI data of AB Aur and TW Hya (see Section 2.4). Both targets were registered and flux calibrated using the induced astrogrid. Approximate coverage maps for the final sequence-combined products are visualized in Figure 3. 

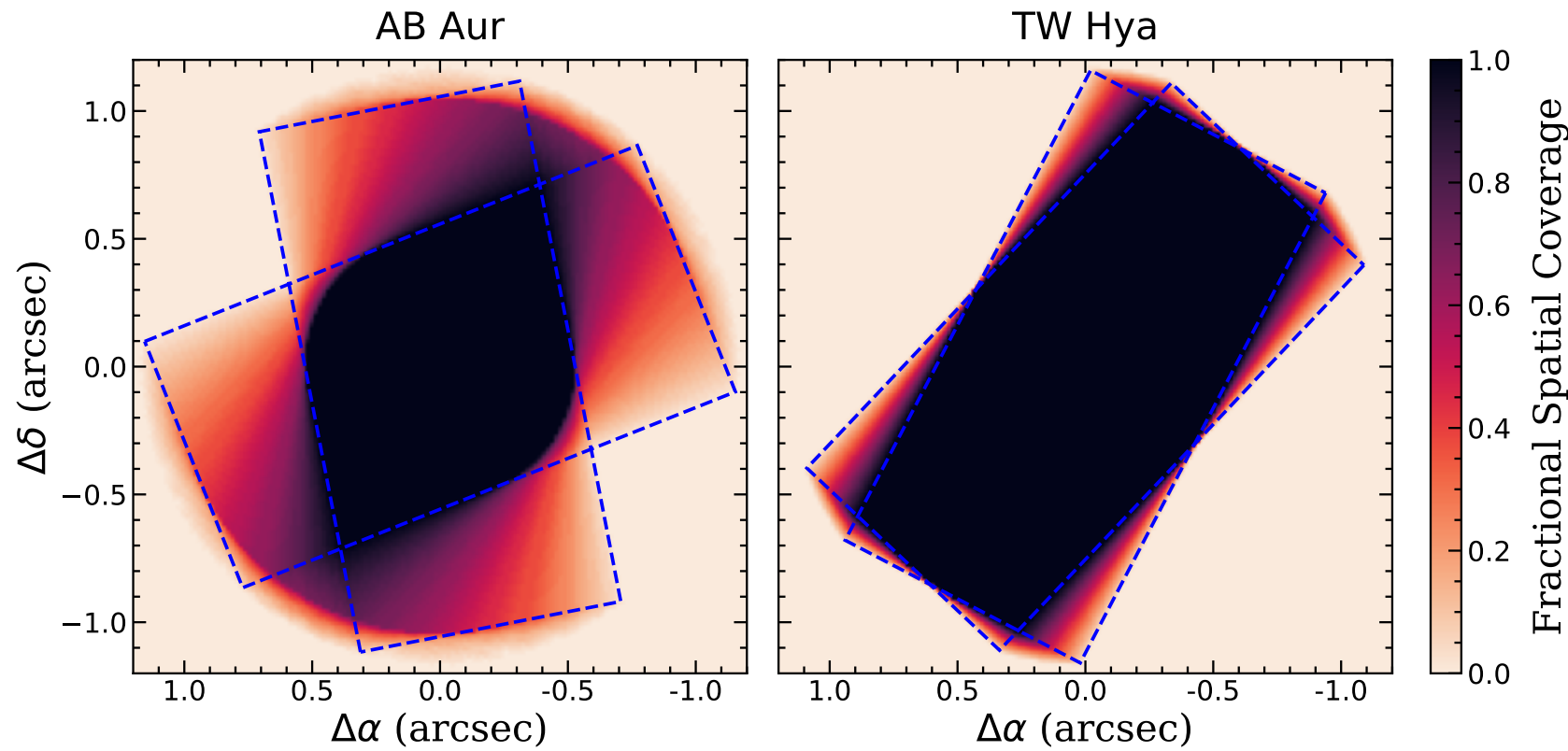

Figure 3. Approximate spatial coverage maps for our sequence-combined AB Aur and TW Hya products. The dashed blue rectangles indicate the extents of the derotated first and last image in each sequence. TW Hya has significantly more area with zero coverage but also more area with full coverage as a result of the TW Hya data's lesser field rotation.

For both targets, a first order instrumental polarization correction was computed within an annulus extending from $r=10$ to $r=30$ pixels $\left(\sim 00^{\prime \prime} 16-0^{\prime \prime} 49\right)$. While significant polarized disk signal is present here, a) this is the case throughout the CHARIS FOV for these targets, and b) this approximation is reasonable only with full azimuthal coverage over the annulus, which occurs for $r \lesssim 30$ pixels $\left(\sim 00^{\prime \prime} 5\right)$ with CHARIS PDI data. However, we note that the inclusion of disk material in the annulus means that this correction is very likely over-estimating the contribution of instrumental polarization; this correction is used only as a placeholder until the formal Mueller Matrix correction is available.

\subsection{AB Aurigae}

Following preprocessing, AB Aurigae observations were matched based on HWP position to produce 16 complete cycles. We then applied the double-differencing PDI technique of Section 3.4 to produce: a) a sequence-combined polarized intensity (PI) image cube (Figure 4), b) J, H, and K-band PI images (Figure 5), and c) a wavelength collapsed broadband PI image (Figure 6). These results show unambiguous detections of the complex spiralarmed disk of AB Aur - not only in wavelength-collapsed products, but also in all 22 individual wavelength channels.

\subsection{TW Hydrae}

HWP matching for TW Hya results in 11 complete cycles. Following the double-differencing procedure, we mask any pixels with coverage in less than $25 \%$ of derotated images to mitigate noisy edges in the products. Figure 7 shows PI images for TW Hya, including a comparison of the $H$-band PI products with and without first-order instrumental polarization correction. $K$-band is not included; the lack of sky frames for this night combined with the weaker $\mathrm{AO}$ correction resulting from the target's faintness produce only a marginal detection of disk features in $K$-band.

\section{CHARIS PDI DISK FORWARD MODELING}

For other varieties of differential imaging (e.g. ADI, RDI, SDI), detailed and computationally intensive forwardmodeling is needed to quantify the (typically significant) erroneous attenuation of disk signal that occurs during 


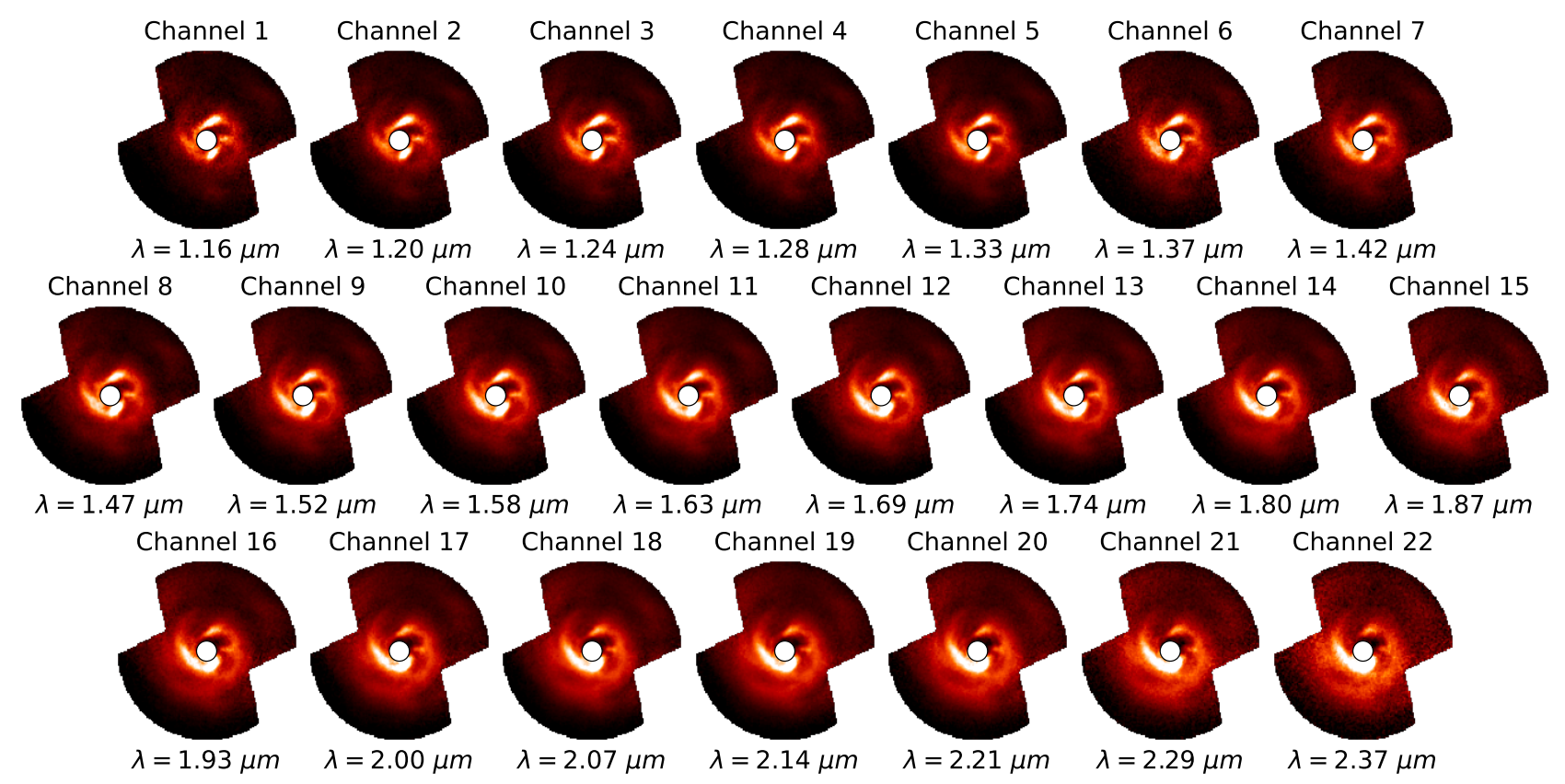

Figure 4. Polarized intensity in 22 wavelength channels for the final, sequence-combined CHARIS PDI-mode observations of $\mathrm{AB}$ Aur. The color stretch is linear throughout, with range chosen arbitrarily for each channel in order to maximize the visibility of disk structures. North is up and east is left, with the FOV extending to $r \sim 1$ 1. 15 .
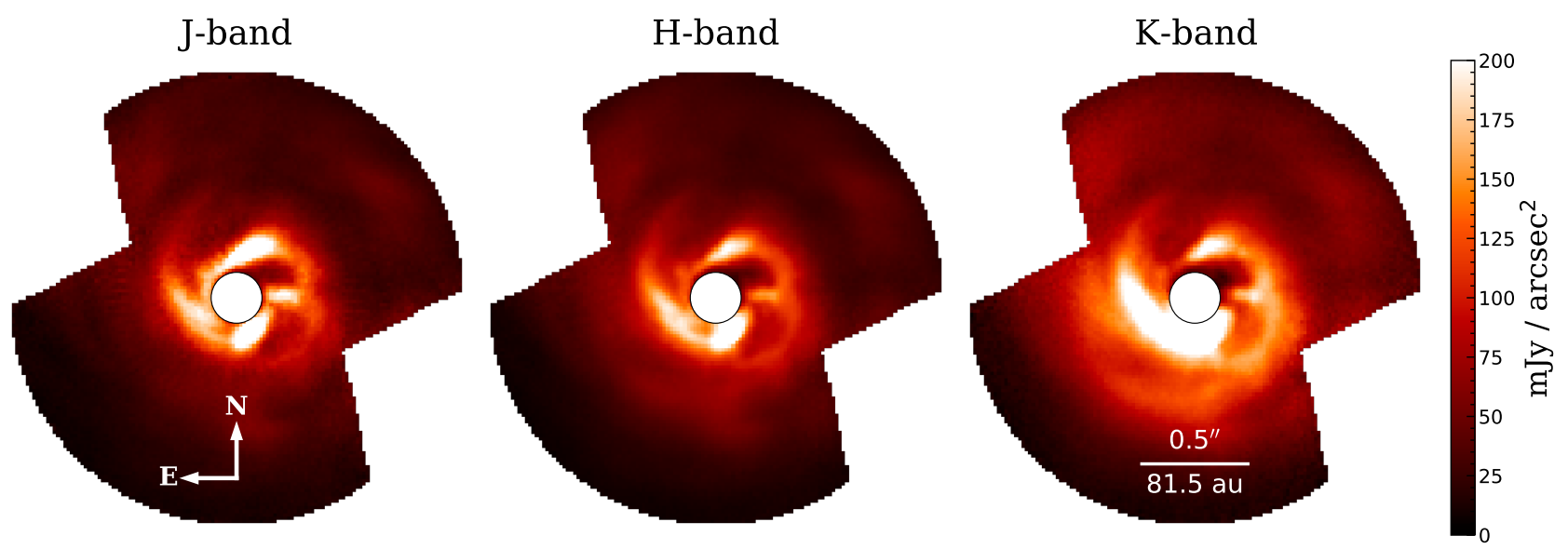

Figure 5. AB Aur CHARIS PI images created by binning sequence-combined $Q$ and $U$ image cubes along the wavelength axis to approximate NIR $J$-band (channels $1-5,1.16-1.33 \mu \mathrm{m}$ ),$H$-band (channels $8-14,1.47-1.80 \mu \mathrm{m}$ ) and $K$-band (channels $16-21,1.93-2.29 \mu \mathrm{m}$ ) images. These $Q$ and $U$ images are then combined to produce PI using equation 6 . 

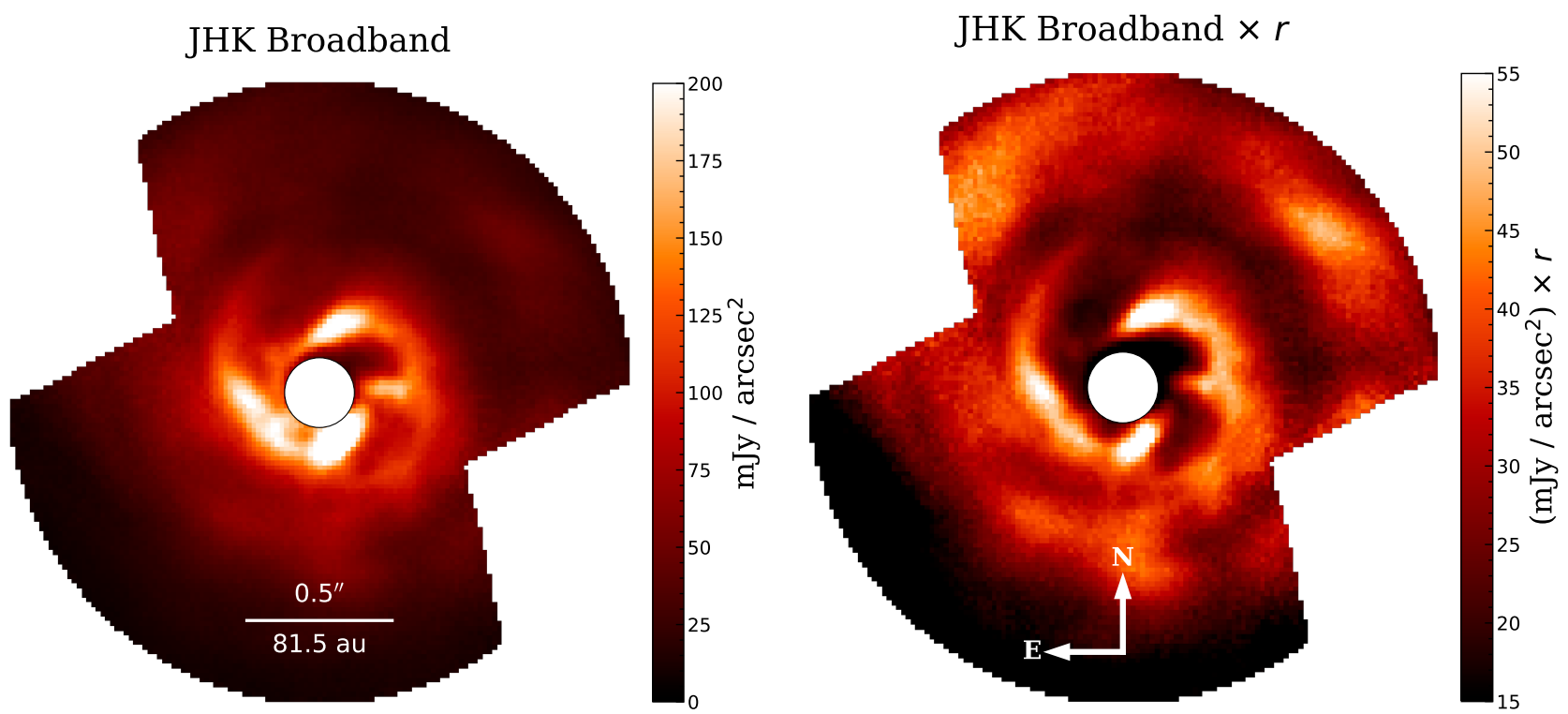

Figure 6. CHARIS wavelength-collapsed PI image of AB Aur created by combining all wavelength channels of the $Q$ and $U$ image cubes before application of equation 6 . The image on the left is presented as the images of Figure 5 , while the image on the right has been multiplied by the projected stellocentric separation in arcsec (assuming an inclination of $30^{\circ}$ and a position angle of $60^{\circ}$ ).

\section{TW Hya, CHARIS Polarized Intensity}
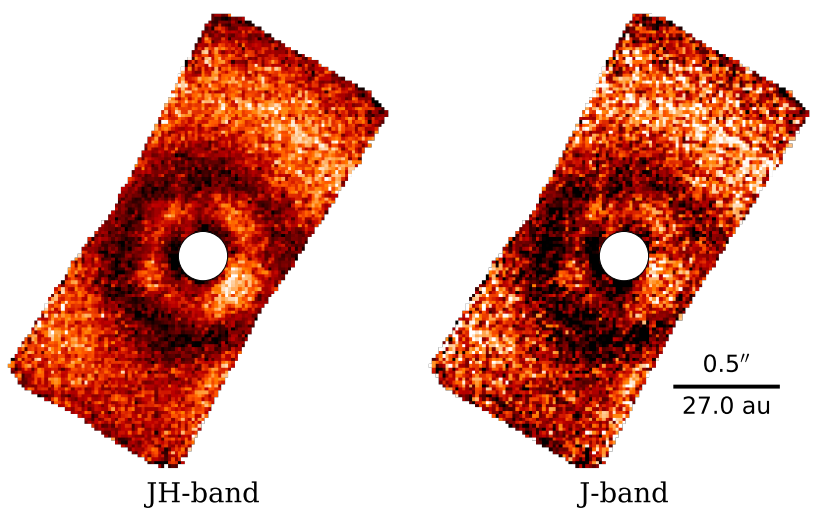

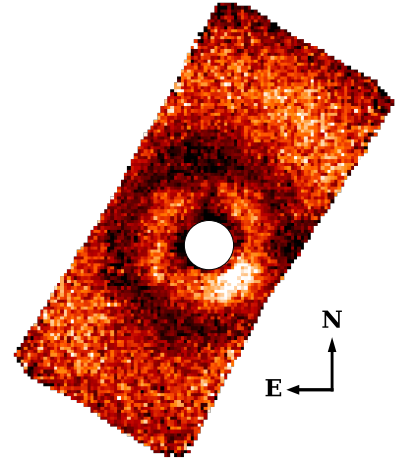

H-band

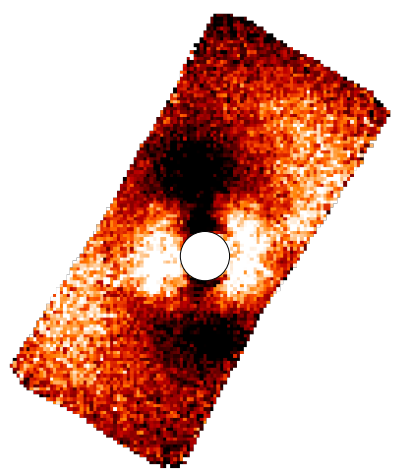

H-band (No IP Correction)

Figure 7. TW Hya CHARIS PI broadband images created by binning sequence-combined $Q$ and $U$ image cubes along the wavelength axis before application of equation 6 . The leftmost image, labeled $J H$-band, is created by combining both $J$-band (channels $1-5,1.16-1.33 \mu \mathrm{m})$ and $H$-band channels $(8-14,1.47-1.80 \mu m)$. The third and fourth images provide a comparison of the result in $H$-band with and without first order instrumental polarization correction. The correction is necessary for a clear detection in this case. 
nulling of the stellar PSF. For PDI, the observed disk image is generally much closer to the true disk, and forward-modeling is relatively simple.

For this purpose, we consider two factors affecting the resulting disk signal:

1. the PSF during our observations

2. the slight difference in orientation between $X^{+}$and $X^{-}$used in double differencing (see Equation 3)

To simulate these effects for a disk model, we proceed as follows. First, synthetic $Q$ and $U$ images are generated for the model. These images are then rotated to match the array of parallactic angles of the appropriate $X^{ \pm}$data (i.e. those of the utilized images at HWP angles of $0^{\circ}$ and $45^{\circ}$ for $Q$ or $22^{\circ} .5$ and 67.5 for $U$ ). This results in two three-dimensional sets of model image sequences. Each frame is then convolved with the empirical PSF model for each wavelength channel (created from either the satellite spots or the unocculted stellar PSF in the data), and frames corresponding to $X^{-}$are multiplied by -1 . Finally, values falling beyond the CHARIS PDI-mode FOV are set to zero. This produces a sequence of image cubes for $Q^{ \pm}$and $U^{ \pm}$comparable to the polarized astrophysical signal contained in the data following single-differencing (Equation 1).

Once synthetic data sequences containing only disk signal have been created, the double-differencing procedure is applied (Equation 3), using the same HWP cycle matches as for the data. Since the orientations of the model images are offset by the same amount as the real data, any effects that result from this will be propagated to the final product from forward-modeling. Following this, the resulting sequences are derotated and combined in the same manner as the data.

Examples of input models alongside corresponding PSF-convolved models and fully processed models resulting from this procedure are shown in Figure 8. These results show little difference between the PSF-convolved and fully processed models. This suggests that, even with the relatively high rate of field rotation for the utilized data, combining exposures at slightly different parallactic angles in double differencing has little effect on the products. This is true even in the case of fine azimuthal features - such as the highly inclined disk model where we should expect the impact to be most significant. Additionally, while we expect no change as a result of the parallactic angle difference in the case of the face-on disk, the change between the PSF-convolved and processed surface brightness (SB) profiles for this model appears comparable with the change for inclined models. Based on this, it seems that the changes we see are predominantly from other sources (e.g. interpolation during image rotation). In general, the impact of the offset in parallactic angle between double-difference pairs will be negligible, but may warrant consideration when observing targets very near zenith.

The relative simplicity of disk forward modeling for PDI data confers a significant strength for disk studies; since the procedure is much less computationally expensive - requiring only image rotation, PSF convolution, and simple algebra - disk models can be forward-modeled orders of magnitude more quickly than for other disk imaging techniques. Combined with swift optimization algorithms, such as differential evolution (DE; see e.g. Ref 14), PDI data enables a much more detailed analysis of disk morphology and composition. This is especially so in the case of debris disks, which can be reasonably approximated with simple scattered-light models (rather than more expensive radiative transfer models). Along with the unique ability of CHARIS to conduct high contrast polarimetry simultaneously at an array of wavelengths, the inherent strength of PDI for disk studies is further magnified.

\section{SUMMARY AND CONCLUSIONS}

The recently-introduced spectropolarimetry mode for the Subaru Telescope's SCExAO/CHARIS provides an exciting new tool for groups studying planet-forming circumstellar disks. This new observing mode enables simultaneous polarized intensity imaging at an array of NIR wavelengths in a $1^{\prime \prime} \times 2^{\prime \prime}$ FOV. We have summarized available instrument configurations and outlined the general procedure used to process CHARIS PDI data. In this procedure, we leverage proven existing software for classical-mode CHARIS data processing alongside purpose-built tools for carrying out HWP matching and double-differencing procedures. With the upcoming implementation of a full instrumental polarization correction, ${ }^{23}$ this new observing mode for SCExAO/CHARIS will enable numerous exciting avenues of study relevant to planet-forming disks. 

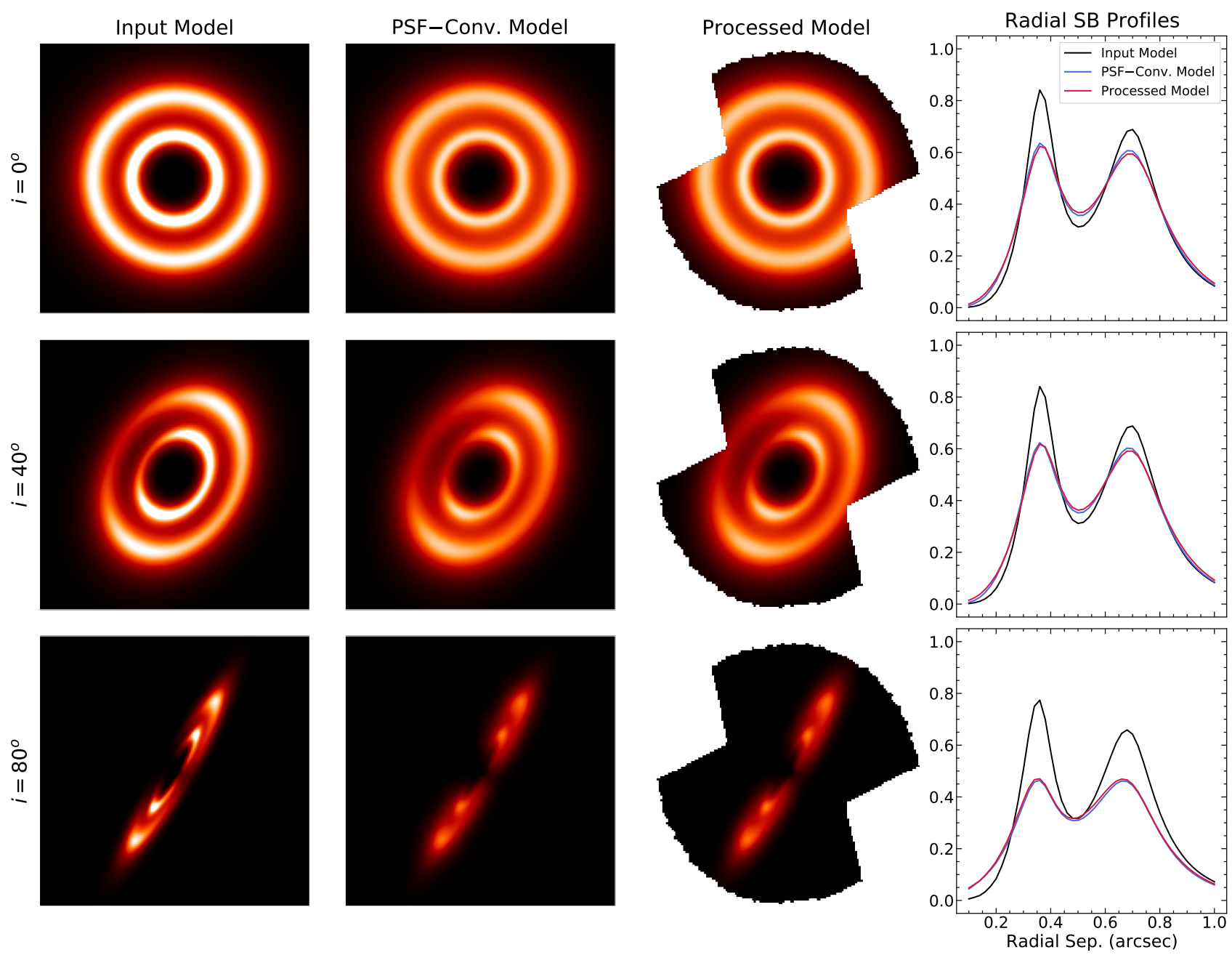

Figure 8. A set of simple synthetic scattered light disk models in polarized intensity are shown throughout the forward modeling procedure for CHARIS PDI-mode. For this purpose, we use the PSF, parallactic angles, and HWP matches from the observations of $\mathrm{AB}$ Aur. Each row corresponds to a distinct input model of differing inclination. The final column displays the radial SB of the images at each stage, measured in apertures of 1 FWHM in diameter and along the major axis of the model. 
Forward-modeling for disks in PDI data, using the simple procedure outlined in Section 5, is extremely time efficient compared to the procedures for other differential imaging techniques. As such, CHARIS PDI-mode will be uniquely well-suited to studying parameters accessible through forward modeling that are expected to have wavelength dependence, such as scattering phase function.

CHARIS PDI-mode will also have utility in helping to understand the sources of spiral arms in disks which exhibit them. From continued observation of such disks, the movement of the features can be measured, which can then be used to discriminate between their two likely causes: yet-unseen companions and gravitational instability. ${ }^{38}$ While total intensity observations might also enable such investigations, it would be much more challenging given that the amount and location of disk attenuation will likely vary between epochs. Being free of significant attenuation, PDI data will significantly strengthen such investigations.

Finally, comparison of polarized intensity and total intensity PDI products (e.g. from application of RDI) enables measurement of fractional polarization (PI/I) across CHARIS's array of wavelengths. These measurements can then be used to identify significant sources of thermal emission relative to other parts of the disk, such as embedded protoplanets, as well as to inform us regarding the properties of the dust within the disk, such as porosity. ${ }^{39}$

\section{ACKNOWLEDGMENTS}

This research is based on data collected at Subaru Telescope, which is operated by the National Astronomical Observatory of Japan. We are honored and grateful for the opportunity of observing the Universe from Maunakea, which has cultural, historical and natural significance in Hawaii.

We wish to acknowledge the critical importance of the current and recent Subaru telescope operators, daycrew, computer support, and office staff employees. Their expertise, ingenuity, and dedication is indispensable to the continued successful operation of Subaru.

The development of SCExAO was supported by the Japan Society for the Promotion of Science (Grant-in-Aid for Research \#23340051, \#26220704, \#23103002, \#19H00703 \& \#19H00695), the Astrobiology Center of the National Institutes of Natural Sciences, Japan, the Mt Cuba Foundation and the director's contingency fund at Subaru Telescope. We acknowledge funding support from the NASA XRP program via grants 80NSSC20K0252 and NNX17AF88G. T.C. was supported by a NASA Senior Postdoctoral Fellowship.

This work has made use of data from the European Space Agency (ESA) mission Gaia (https://www. cosmos.esa.int/gaia), processed by the Gaia Data Processing and Analysis Consortium (DPAC, https:// www.cosmos.esa.int/web/gaia/dpac/consortium). Funding for the DPAC has been provided by national institutions, in particular the institutions participating in the Gaia Multilateral Agreement.

\section{REFERENCES}

[1] Beuzit, J. L., Vigan, A., Mouillet, D., Dohlen, K., Gratton, R., Boccaletti, A., Sauvage, J. F., Schmid, H. M., Langlois, M., Petit, C., Baruffolo, A., Feldt, M., Milli, J., Wahhaj, Z., Abe, L., Anselmi, U., Antichi, J., Barette, R., Baudrand, J., Baudoz, P., Bazzon, A., Bernardi, P., Blanchard, P., Brast, R., Bruno, P., Buey, T., Carbillet, M., Carle, M., Cascone, E., Chapron, F., Charton, J., Chauvin, G., Claudi, R., Costille, A., De Caprio, V., de Boer, J., Delboulbé, A., Desidera, S., Dominik, C., Downing, M., Dupuis, O., Fabron, C., Fantinel, D., Farisato, G., Feautrier, P., Fedrigo, E., Fusco, T., Gigan, P., Ginski, C., Girard, J., Giro, E., Gisler, D., Gluck, L., Gry, C., Henning, T., Hubin, N., Hugot, E., Incorvaia, S., Jaquet, M., Kasper, M., Lagadec, E., Lagrange, A. M., Le Coroller, H., Le Mignant, D., Le Ruyet, B., Lessio, G., Lizon, J. L., Llored, M., Lundin, L., Madec, F., Magnard, Y., Marteaud, M., Martinez, P., Maurel, D., Ménard, F., Mesa, D., Möller-Nilsson, O., Moulin, T., Moutou, C., Origné, A., Parisot, J., Pavlov, A., Perret, D., Pragt, J., Puget, P., Rabou, P., Ramos, J., Reess, J. M., Rigal, F., Rochat, S., Roelfsema, R., Rousset, G., Roux, A., Saisse, M., Salasnich, B., Santambrogio, E., Scuderi, S., Segransan, D., Sevin, A., Siebenmorgen, R., Soenke, C., Stadler, E., Suarez, M., Tiphène, D., Turatto, M., Udry, S., Vakili, F., Waters, L. B. F. M., Weber, L., Wildi, F., Zins, G., and Zurlo, A., "SPHERE: the exoplanet imager for the Very Large Telescope," Astronomy $\mathcal{E}$ Astrophysics 631, A155 (Nov. 2019). 
[2] Macintosh, B., Graham, J. R., Barman, T., De Rosa, R. J., Konopacky, Q., Marley, M. S., Marois, C., Nielsen, E. L., Pueyo, L., Rajan, A., Rameau, J., Saumon, D., Wang, J. J., Patience, J., Ammons, M., Arriaga, P., Artigau, E., Beckwith, S., Brewster, J., Bruzzone, S., Bulger, J., Burningham, B., Burrows, A. S., Chen, C., Chiang, E., Chilcote, J. K., Dawson, R. I., Dong, R., Doyon, R., Draper, Z. H., Duchêne, G., Esposito, T. M., Fabrycky, D., Fitzgerald, M. P., Follette, K. B., Fortney, J. J., Gerard, B., Goodsell, S., Greenbaum, A. Z., Hibon, P., Hinkley, S., Cotten, T. H., Hung, L. W., Ingraham, P., Johnson-Groh, M., Kalas, P., Lafreniere, D., Larkin, J. E., Lee, J., Line, M., Long, D., Maire, J., Marchis, F., Matthews, B. C., Max, C. E., Metchev, S., Millar-Blanchaer, M. A., Mittal, T., Morley, C. V., Morzinski, K. M., Murray-Clay, R., Oppenheimer, R., Palmer, D. W., Patel, R., Perrin, M. D., Poyneer, L. A., Rafikov, R. R., Rantakyrö, F. T., Rice, E. L., Rojo, P., Rudy, A. R., Ruffio, J. B., Ruiz, M. T., Sadakuni, N., Saddlemyer, L., Salama, M., Savransky, D., Schneider, A. C., Sivaramakrishnan, A., Song, I., Soummer, R., Thomas, S., Vasisht, G., Wallace, J. K., Ward-Duong, K., Wiktorowicz, S. J., Wolff, S. G., and Zuckerman, B., "Discovery and spectroscopy of the young jovian planet 51 Eri b with the Gemini Planet Imager," Science 350, 64-67 (Oct 2015).

[3] Jovanovic, N., Martinache, F., Guyon, O., Clergeon, C., Singh, G., Kudo, T., Garrel, V., Newman, K., Doughty, D., Lozi, J., Males, J., Minowa, Y., Hayano, Y., Takato, N., Morino, J., Kuhn, J., Serabyn, E., Norris, B., Tuthill, P., Schworer, G., Stewart, P., Close, L., Huby, E., Perrin, G., Lacour, S., Gauchet, L., Vievard, S., Murakami, N., Oshiyama, F., Baba, N., Matsuo, T., Nishikawa, J., Tamura, M., Lai, O., Marchis, F., Duchene, G., Kotani, T., and Woillez, J., "The Subaru Coronagraphic Extreme Adaptive Optics System: Enabling High-Contrast Imaging on Solar-System Scales," Publications of the Astronomical Society of the Pacific 127, 890 (Sep 2015).

[4] Lozi, J., Guyon, O., Jovanovic, N., Goebel, S., Pathak, P., Skaf, N., Sahoo, A., Norris, B., Martinache, F., N'Diaye, M., Mazin, B., Walter, A. B., Tuthill, P., Kudo, T., Kawahara, H., Kotani, T., Ireland, M., Cvetojevic, N., Huby, E., Lacour, S., Vievard, S., Groff, T. D., Chilcote, J. K., Kasdin, J., Knight, J., Snik, F., Doelman, D., Minowa, Y., Clergeon, C., Takato, N., Tamura, M., Currie, T., Takami, H., and Hayashi, M., "SCExAO, an instrument with a dual purpose: perform cutting-edge science and develop new technologies," in [Proc. SPIE.], Society of Photo-Optical Instrumentation Engineers (SPIE) Conference Series 10703, 1070359 (July 2018).

[5] Currie, T., Guyon, O., Lozi, J., Sahoo, A., Vievard, S., Deo, V., Chilcote, J., Groff, T., Brandt, T. D., Lawson, K., Skaf, N., Martinache, F., and Kasdin, N. J., "On-sky performance and recent results from the Subaru coronagraphic extreme adaptive optics system," in [Society of Photo-Optical Instrumentation Engineers (SPIE) Conference Series], Society of Photo-Optical Instrumentation Engineers (SPIE) Conference Series 11448, 114487H (Dec. 2020).

[6] Lagrange, A.-M., Bonnefoy, M., Chauvin, G., Apai, D., Ehrenreich, D., Boccaletti, A., Gratadour, D., Rouan, D., Mouillet, D., Lacour, S., and Kasper, M., "A Giant Planet Imaged in the Disk of the Young Star $\beta$ Pictoris," Science 329, 57 (July 2010).

[7] Keppler, M., Benisty, M., Müller, A., Henning, T., van Boekel, R., Cantalloube, F., Ginski, C., van Holstein, R. G., Maire, A. L., Pohl, A., Samland, M., Avenhaus, H., Baudino, J. L., Boccaletti, A., de Boer, J., Bonnefoy, M., Chauvin, G., Desidera, S., Langlois, M., Lazzoni, C., Marleau, G. D., Mordasini, C., Pawellek, N., Stolker, T., Vigan, A., Zurlo, A., Birnstiel, T., Brandner, W., Feldt, M., Flock, M., Girard, J., Gratton, R., Hagelberg, J., Isella, A., Janson, M., Juhasz, A., Kemmer, J., Kral, Q., Lagrange, A. M., Launhardt, R., Matter, A., Ménard, F., Milli, J., Mollière, P., Olofsson, J., Pérez, L., Pinilla, P., Pinte, C., Quanz, S. P., Schmidt, T., Udry, S., Wahhaj, Z., Williams, J. P., Buenzli, E., Cudel, M., Dominik, C., Galicher, R., Kasper, M., Lannier, J., Mesa, D., Mouillet, D., Peretti, S., Perrot, C., Salter, G., Sissa, E., Wildi, F., Abe, L., Antichi, J., Augereau, J. C., Baruffolo, A., Baudoz, P., Bazzon, A., Beuzit, J. L., Blanchard, P., Brems, S. S., Buey, T., De Caprio, V., Carbillet, M., Carle, M., Cascone, E., Cheetham, A., Claudi, R., Costille, A., Delboulbé, A., Dohlen, K., Fantinel, D., Feautrier, P., Fusco, T., Giro, E., Gluck, L., Gry, C., Hubin, N., Hugot, E., Jaquet, M., Le Mignant, D., Llored, M., Madec, F., Magnard, Y., Martinez, P., Maurel, D., Meyer, M., Möller-Nilsson, O., Moulin, T., Mugnier, L., Origné, A., Pavlov, A., Perret, D., Petit, C., Pragt, J., Puget, P., Rabou, P., Ramos, J., Rigal, F., Rochat, S., Roelfsema, R., Rousset, G., Roux, A., Salasnich, B., Sauvage, J. F., Sevin, A., Soenke, C., Stadler, E., Suarez, M., Turatto, M., and 
Weber, L., "Discovery of a planetary-mass companion within the gap of the transition disk around PDS 70," Astronomy \& Astrophysics 617, A44 (Sept. 2018).

[8] Marois, C., Lafrenière, D., Doyon, R., Macintosh, B., and Nadeau, D., "Angular Differential Imaging: A Powerful High-Contrast Imaging Technique," Astrophysical Journal 641, 556-564 (Apr. 2006).

[9] Sparks, W. B. and Ford, H. C., "Imaging Spectroscopy for Extrasolar Planet Detection," Astrophysical Journal 578, 543-564 (Oct. 2002).

[10] Pueyo, L., "Detection and Characterization of Exoplanets using Projections on Karhunen Loeve Eigenimages: Forward Modeling," Astrophysical Journal 824, 117 (June 2016).

[11] Currie, T., Guyon, O., Lozi, J., Groff, T., Kasdin, N. J., Martinache, F., Brandt, T. D., Chilcote, J., Marois, C., Gerard, B., Jovanovic, N., and Vievard, S., "Performance and early science with the Subaru Coronagraphic Extreme Adaptive Optics project," in [Techniques and Instrumentation for Detection of Exoplanets IX], Shaklan, S. B., ed., 11117, 285 - 300, International Society for Optics and Photonics, SPIE (2019).

[12] Rich, E. A., Wisniewski, J. P., Currie, T., Fukagawa, M., Grady, C. A., Sitko, M. L., Pikhartova, M., Hashimoto, J., Abe, L., Brandner, W., Brandt, T. D., Carson, J. C., Chilcote, J., Dong, R., Feldt, M., Goto, M., Groff, T., Guyon, O., Hayano, Y., Hayashi, M., Hayashi, S. S., Henning, T., Hodapp, K. W., Ishii, M., Iye, M., Janson, M., Jovanovic, N., Kandori, R., Kasdin, J., Knapp, G. R., Kudo, T., Kusakabe, N., Kuzuhara, M., Kwon, J., Lozi, J., Martinache, F., Matsuo, T., Mayama, S., McElwain, M. W., Miyama, S., Morino, J.-I., Moro-Martin, A., Nakagawa, T., Nishimura, T., Pyo, T.-S., Serabyn, E., Suto, H., Russel, R. W., Suzuki, R., Takami, M., Takato, N., Terada, H., Thalmann, C., Turner, E. L., Uyama, T., Wagner, K. R., Watanabe, M., Yamada, T., Takami, H., Usuda, T., and Tamura, M., "Multi-epoch Direct Imaging and Time-variable Scattered Light Morphology of the HD 163296 Protoplanetary Disk," Astrophysical Journal 875, 38 (Apr. 2019).

[13] Currie, T., Marois, C., Cieza, L., Mulders, G. D., Lawson, K., Caceres, C., Rodriguez-Ruiz, D., Wisniewski, J., Guyon, O., Brandt, T. D., Kasdin, N. J., Groff, T. D., Lozi, J., Chilcote, J., Hodapp, K., Jovanovic, N., Martinache, F., Skaf, N., Lyra, W., Tamura, M., Asensio-Torres, R., Dong, R., Grady, C., Gerard, B., Fukagawa, M., Hand, D., Hayashi, M., Henning, T., Kudo, T., Kuzuhara, M., Kwon, J., McElwain, M. W., and Uyama, T., "No Clear, Direct Evidence for Multiple Protoplanets Orbiting LkCa 15: LkCa 15 bcd are Likely Inner Disk Signals," Astrophysical Journal Letters 877, L3 (May 2019).

[14] Lawson, K., Currie, T., Wisniewski, J. P., Tamura, M., Schneider, G., Augereau, J.-C., Brandt, T. D., Guyon, O., Kasdin, N. J., Groff, T. D., Lozi, J., Chilcote, J., Hodapp, K., Jovanovic, N., Martinache, F., Skaf, N., Akiyama, E., Henning, T., Knapp, G. R., Kwon, J., Mayama, S., McElwain, M. W., Sitko, M. L., Asensio-Torres, R., Uyama, T., and Wagner, K., "SCExAO/CHARIS Near-infrared Integral Field Spectroscopy of the HD 15115 Debris Disk," Astronomical Journal 160, 163 (Oct. 2020).

[15] Perrin, M. D., Duchene, G., Millar-Blanchaer, M., Fitzgerald, M. P., Graham, J. R., Wiktorowicz, S. J., Kalas, P. G., Macintosh, B., Bauman, B., Cardwell, A., Chilcote, J., De Rosa, R. J., Dillon, D., Doyon, R., Dunn, J., Erikson, D., Gavel, D., Goodsell, S., Hartung, M., Hibon, P., Ingraham, P., Kerley, D., Konapacky, Q., Larkin, J. E., Maire, J., Marchis, F., Marois, C., Mittal, T., Morzinski, K. M., Oppenheimer, B. R., Palmer, D. W., Patience, J., Poyneer, L., Pueyo, L., Rantakyrö, F. T., Sadakuni, N., Saddlemyer, L., Savransky, D., Soummer, R., Sivaramakrishnan, A., Song, I., Thomas, S., Wallace, J. K., Wang, J. J., and Wolff, S. G., "Polarimetry with the Gemini Planet Imager: Methods, Performance at First Light, and the Circumstellar Ring around HR 4796A," Astrophysical Journal 799, 182 (Feb. 2015).

[16] de Boer, J., Langlois, M., van Holstein, R. G., Girard, J. H., Mouillet, D., Vigan, A., Dohlen, K., Snik, F., Keller, C. U., Ginski, C., Stam, D. M., Milli, J., Wahhaj, Z., Kasper, M., Schmid, H. M., Rabou, P., Gluck, L., Hugot, E., Perret, D., Martinez, P., Weber, L., Pragt, J., Sauvage, J. F., Boccaletti, A., Le Coroller, H., Dominik, C., Henning, T., Lagadec, E., Ménard, F., Turatto, M., Udry, S., Chauvin, G., Feldt, M., and Beuzit, J. L., "Polarimetric imaging mode of VLT/SPHERE/IRDIS. I. Description, data reduction, and observing strategy," Astronomy \&3 Astrophysics 633, A63 (Jan. 2020).

[17] van Holstein, R. G., Girard, J. H., de Boer, J., Snik, F., Milli, J., Stam, D. M., Ginski, C., Mouillet, D., Wahhaj, Z., Schmid, H. M., Keller, C. U., Langlois, M., Dohlen, K., Vigan, A., Pohl, A., Carbillet, M., Fantinel, D., Maurel, D., Origné, A., Petit, C., Ramos, J., Rigal, F., Sevin, A., Boccaletti, A., Le Coroller, H., Dominik, C., Henning, T., Lagadec, E., Ménard, F., Turatto, M., Udry, S., Chauvin, G., Feldt, M., and 
Beuzit, J. L., "Polarimetric imaging mode of VLT/SPHERE/IRDIS. II. Characterization and correction of instrumental polarization effects," Astronomy \&3 Astrophysics 633, A64 (Jan. 2020).

[18] Suzuki, R., Kudo, T., Hashimoto, J., Carson, J., Egner, S., Goto, M., Hattori, M., Hayano, Y., Hodapp, K., Ito, M., Iye, M., Jacobson, S., Kandori, R., Kusakabe, N., Kuzuhara, M., Matsuo, T., Mcelwain, M., Morino, J.-I., Oya, S., Saito, Y., Shelton, R., Stahlberger, V., Suto, H., Takami, H., Thalmann, C., Watanabe, M., Yamada, H., and Tamura, M., "Performance characterization of the HiCIAO instrument for the Subaru Telescope," in [Ground-based and Airborne Instrumentation for Astronomy III], McLean, I. S., Ramsay, S. K., and Takami, H., eds., Society of Photo-Optical Instrumentation Engineers (SPIE) Conference Series 7735, 773530 (July 2010).

[19] Perrin, M. D., Schneider, G., Duchene, G., Pinte, C., Grady, C. A., Wisniewski, J. P., and Hines, D. C., "The Case of AB Aurigae's Disk in Polarized Light: Is there Truly a Gap?," Astrophysical Journal Letters 707, L132-L136 (Dec. 2009).

[20] Uyama, T., Hashimoto, J., Kuzuhara, M., Mayama, S., Akiyama, E., Currie, T., Livingston, J., Kudo, T., Kusakabe, N., Abe, L., Brandner, W., Brandt, T. D., Carson, J. C., Egner, S., Feldt, M., Goto, M., Grady, C. A., Guyon, O., Hayano, Y., Hayashi, M., Hayashi, S. S., Henning, T., Hodapp, K. W., Ishii, M., Iye, M., Janson, M., Kandori, R., Knapp, G. R., Kwon, J., Matsuo, T., Mcelwain, M. W., Miyama, S., Morino, J.-I., Moro-Martin, A., Nishimura, T., Pyo, T.-S., Serabyn, E., Suenaga, T., Suto, H., Suzuki, R., Takahashi, Y. H., Takami, M., Takato, N., Terada, H., Thalmann, C., Turner, E. L., Watanabe, M., Wisniewski, J., Yamada, T., Takami, H., Usuda, T., and Tamura, M., "The SEEDS High-Contrast Imaging Survey of Exoplanets Around Young Stellar Objects," Astronomical Journal 153, 106 (Mar. 2017).

[21] Groff, T. D., Chilcote, J., Kasdin, N. J., Galvin, M., Loomis, C., Carr, M. A., Brand t, T., Knapp, G., Limbach, M. A., Guyon, O., Jovanovic, N., McElwain, M. W., Takato, N., and Hayashi, M., "Laboratory testing and performance verification of the CHARIS integral field spectrograph," in [Ground-based and Airborne Instrumentation for Astronomy VI], Society of Photo-Optical Instrumentation Engineers (SPIE) Conference Series 9908, 990800 (Aug 2016).

[22] Lozi, J., Guyon, O., Jovanovic, N., Norris, B., Groff, T., Chilcote, J., Kasdin, N., Kudo, T., Tamura, M., Zhang, J., Bos, S., Snik, F., Doelman, D., Vievard, S., Sahoo, A., Currie, T., and Martinache, F., "New NIR spectro-polarimetric modes for the SCExAO instrument," in [American Astronomical Society Meeting Abstracts \#235], American Astronomical Society Meeting Abstracts 235, 161.07 (Jan. 2020).

[23] van Holstein, R. G., Bos, S. P., Ruigrok, J., Lozi, J., Guyon, O., Norris, B., Snik, F., Chilcote, J., Currie, T., Groff, T. D., 't Hart, J., Jovanovic, N., Kasdin, J., Kudo, T., Martinache, F., Mazin, B., Sahoo, A., Tamura, M., Vievard, S., Walter, A., and Zhang, J., "Calibration of the instrumental polarization effects of SCExAO-CHARIS' spectropolarimetric mode," in [Society of Photo-Optical Instrumentation Engineers (SPIE) Conference Series], Society of Photo-Optical Instrumentation Engineers (SPIE) Conference Series 11447, 114475B (Dec. 2020).

[24] Kenyon, S. J. and Bromley, B. C., "Variations on Debris Disks: Icy Planet Formation at 30-150 AU for 1-3 $\mathrm{M}_{\odot}$ Main-Sequence Stars," Astrophysical Journal Supplements 179, 451-483 (Dec 2008).

[25] Gaia Collaboration, Prusti, T., de Bruijne, J. H. J., Brown, A. G. A., Vallenari, A., Babusiaux, C., BailerJones, C. A. L., Bastian, U., Biermann, M., Evans, D. W., Eyer, L., Jansen, F., Jordi, C., Klioner, S. A., Lammers, U., Lindegren, L., Luri, X., Mignard, F., Milligan, D. J., Panem, C., Poinsignon, V., Pourbaix, D., Randich, S., Sarri, G., Sartoretti, P., Siddiqui, H. I., Soubiran, C., Valette, V., van Leeuwen, F., Walton, N. A., Aerts, C., Arenou, F., Cropper, M., Drimmel, R., Høg, E., Katz, D., Lattanzi, M. G., O'Mullane, W., Grebel, E. K., Holland, A. D., Huc, C., Passot, X., Bramante, L., Cacciari, C., Castañeda, J., Chaoul, L., Cheek, N., De Angeli, F., Fabricius, C., Guerra, R., Hernández, J., Jean-Antoine-Piccolo, A., Masana, E., Messineo, R., Mowlavi, N., Nienartowicz, K., Ordóñez-Blanco, D., Panuzzo, P., Portell, J., Richards, P. J., Riello, M., Seabroke, G. M., Tanga, P., Thévenin, F., Torra, J., Els, S. G., Gracia-Abril, G., Comoretto, G., Garcia-Reinaldos, M., Lock, T., Mercier, E., Altmann, M., Andrae, R., Astraatmadja, T. L., BellasVelidis, I., Benson, K., Berthier, J., Blomme, R., Busso, G., Carry, B., Cellino, A., Clementini, G., Cowell, S., Creevey, O., Cuypers, J., Davidson, M., De Ridder, J., de Torres, A., Delchambre, L., Dell'Oro, A., Ducourant, C., Frémat, Y., García-Torres, M., Gosset, E., Halbwachs, J. L., Hambly, N. C., Harrison, D. L., Hauser, M., Hestroffer, D., Hodgkin, S. T., Huckle, H. E., Hutton, A., Jasniewicz, G., Jordan, S., Kontizas, M., Korn, A. J., Lanzafame, A. C., Manteiga, M., Moitinho, A., Muinonen, K., Osinde, J., 
Pancino, E., Pauwels, T., Petit, J. M., Recio-Blanco, A., Robin, A. C., Sarro, L. M., Siopis, C., Smith, M., Smith, K. W., Sozzetti, A., Thuillot, W., van Reeven, W., Viala, Y., Abbas, U., Abreu Aramburu, A., Accart, S., Aguado, J. J., Allan, P. M., Allasia, W., Altavilla, G., Álvarez, M. A., Alves, J., Anderson, R. I., Andrei, A. H., Anglada Varela, E., Antiche, E., Antoja, T., Antón, S., Arcay, B., Atzei, A., Ayache, L., Bach, N., Baker, S. G., Balaguer-Núñez, L., Barache, C., Barata, C., Barbier, A., Barblan, F., Baroni, M., Barrado y Navascués, D., Barros, M., Barstow, M. A., Becciani, U., Bellazzini, M., Bellei, G., Bello García, A., Belokurov, V., Bendjoya, P., Berihuete, A., Bianchi, L., Bienaymé, O., Billebaud, F., Blagorodnova, N., Blanco-Cuaresma, S., Boch, T., Bombrun, A., Borrachero, R., Bouquillon, S., Bourda, G., Bouy, H., Bragaglia, A., Breddels, M. A., Brouillet, N., Brüsemeister, T., Bucciarelli, B., Budnik, F., Burgess, P., Burgon, R., Burlacu, A., Busonero, D., Buzzi, R., Caffau, E., Cambras, J., Campbell, H., Cancelliere, R., Cantat-Gaudin, T., Carlucci, T., Carrasco, J. M., Castellani, M., Charlot, P., Charnas, J., Charvet, P., Chassat, F., Chiavassa, A., Clotet, M., Cocozza, G., Collins, R. S., Collins, P., Costigan, G., Crifo, F., Cross, N. J. G., Crosta, M., Crowley, C., Dafonte, C., Damerdji, Y., Dapergolas, A., David, P., David, M., De Cat, P., de Felice, F., de Laverny, P., De Luise, F., De March, R., de Martino, D., de Souza, R., Debosscher, J., del Pozo, E., Delbo, M., Delgado, A., Delgado, H. E., di Marco, F., Di Matteo, P., Diakite, S., Distefano, E., Dolding, C., Dos Anjos, S., Drazinos, P., Durán, J., Dzigan, Y., Ecale, E., Edvardsson, B., Enke, H., Erdmann, M., Escolar, D., Espina, M., Evans, N. W., Eynard Bontemps, G., Fabre, C., Fabrizio, M., Faigler, S., Falcão, A. J., Farràs Casas, M., Faye, F., Federici, L., Fedorets, G., Fernández-Hernández, J., Fernique, P., Fienga, A., Figueras, F., Filippi, F., Findeisen, K., Fonti, A., Fouesneau, M., Fraile, E., Fraser, M., Fuchs, J., Furnell, R., Gai, M., Galleti, S., Galluccio, L., Garabato, D., García-Sedano, F., Garé, P., Garofalo, A., Garralda, N., Gavras, P., Gerssen, J., Geyer, R., Gilmore, G., Girona, S., Giuffrida, G., Gomes, M., González-Marcos, A., González-Núñez, J., González-Vidal, J. J., Granvik, M., Guerrier, A., Guillout, P., Guiraud, J., Gúrpide, A., Gutiérrez-Sánchez, R., Guy, L. P., Haigron, R., Hatzidimitriou, D., Haywood, M., Heiter, U., Helmi, A., Hobbs, D., Hofmann, W., Holl, B., Holland, G., Hunt, J. A. S., Hypki, A., Icardi, V., Irwin, M., Jevardat de Fombelle, G., Jofré, P., Jonker, P. G., Jorissen, A., Julbe, F., Karampelas, A., Kochoska, A., Kohley, R., Kolenberg, K., Kontizas, E., Koposov, S. E., Kordopatis, G., Koubsky, P., Kowalczyk, A., Krone-Martins, A., Kudryashova, M., Kull, I., Bachchan, R. K., Lacoste-Seris, F., Lanza, A. F., Lavigne, J. B., Le Poncin-Lafitte, C., Lebreton, Y., Lebzelter, T., Leccia, S., Leclerc, N., Lecoeur-Taibi, I., Lemaitre, V., Lenhardt, H., Leroux, F., Liao, S., Licata, E., Lindstrøm, H. E. P., Lister, T. A., Livanou, E., Lobel, A., Löffler, W., López, M., Lopez-Lozano, A., Lorenz, D., Loureiro, T., MacDonald, I., Magalhães Fernandes, T., Managau, S., Mann, R. G., Mantelet, G., Marchal, O., Marchant, J. M., Marconi, M., Marie, J., Marinoni, S., Marrese, P. M., Marschalkó, G., Marshall, D. J., Martín-Fleitas, J. M., Martino, M., Mary, N., Matijevič, G., Mazeh, T., McMillan, P. J., Messina, S., Mestre, A., Michalik, D., Millar, N. R., Miranda, B. M. H., Molina, D., Molinaro, R., Molinaro, M., Molnár, L., Moniez, M., Montegriffo, P., Monteiro, D., Mor, R., Mora, A., Morbidelli, R., Morel, T., Morgenthaler, S., Morley, T., Morris, D., Mulone, A. F., Muraveva, T., Musella, I., Narbonne, J., Nelemans, G., Nicastro, L., Noval, L., Ordénovic, C., Ordieres-Meré, J., Osborne, P., Pagani, C., Pagano, I., Pailler, F., Palacin, H., Palaversa, L., Parsons, P., Paulsen, T., Pecoraro, M., Pedrosa, R., Pentikäinen, H., Pereira, J., Pichon, B., Piersimoni, A. M., Pineau, F. X., Plachy, E., Plum, G., Poujoulet, E., Prša, A., Pulone, L., Ragaini, S., Rago, S., Rambaux, N., Ramos-Lerate, M., Ranalli, P., Rauw, G., Read, A., Regibo, S., Renk, F., Reylé, C., Ribeiro, R. A., Rimoldini, L., Ripepi, V., Riva, A., Rixon, G., Roelens, M., Romero-Gómez, M., Rowell, N., Royer, F., Rudolph, A., Ruiz-Dern, L., Sadowski, G., Sagristà Sellés, T., Sahlmann, J., Salgado, J., Salguero, E., Sarasso, M., Savietto, H., Schnorhk, A., Schultheis, M., Sciacca, E., Segol, M., Segovia, J. C., Segransan, D., Serpell, E., Shih, I. C., Smareglia, R., Smart, R. L., Smith, C., Solano, E., Solitro, F., Sordo, R., Soria Nieto, S., Souchay, J., Spagna, A., Spoto, F., Stampa, U., Steele, I. A., Steidelmüller, H., Stephenson, C. A., Stoev, H., Suess, F. F., Süveges, M., Surdej, J., Szabados, L., Szegedi-Elek, E., Tapiador, D., Taris, F., Tauran, G., Taylor, M. B., Teixeira, R., Terrett, D., Tingley, B., Trager, S. C., Turon, C., Ulla, A., Utrilla, E., Valentini, G., van Elteren, A., Van Hemelryck, E., van Leeuwen, M., Varadi, M., Vecchiato, A., Veljanoski, J., Via, T., Vicente, D., Vogt, S., Voss, H., Votruba, V., Voutsinas, S., Walmsley, G., Weiler, M., Weingrill, K., Werner, D., Wevers, T., Whitehead, G., Wyrzykowski, Ł., Yoldas, A., Žerjal, M., Zucker, S., Zurbach, C., Zwitter, T., Alecu, A., Allen, M., Allende Prieto, C., Amorim, A., Anglada-Escudé, G., Arsenijevic, V., Azaz, S., Balm, P., Beck, M., Bernstein, H. H., Bigot, L., Bijaoui, A., Blasco, C., Bonfigli, M., Bono, G., Boudreault, 
S., Bressan, A., Brown, S., Brunet, P. M., Bunclark, P., Buonanno, R., Butkevich, A. G., Carret, C., Carrion, C., Chemin, L., Chéreau, F., Corcione, L., Darmigny, E., de Boer, K. S., de Teodoro, P., de Zeeuw, P. T., Delle Luche, C., Domingues, C. D., Dubath, P., Fodor, F., Frézouls, B., Fries, A., Fustes, D., Fyfe, D., Gallardo, E., Gallegos, J., Gardiol, D., Gebran, M., Gomboc, A., Gómez, A., Grux, E., Gueguen, A., Heyrovsky, A., Hoar, J., Iannicola, G., Isasi Parache, Y., Janotto, A. M., Joliet, E., Jonckheere, A., Keil, R., Kim, D. W., Klagyivik, P., Klar, J., Knude, J., Kochukhov, O., Kolka, I., Kos, J., Kutka, A., Lainey, V., LeBouquin, D., Liu, C., Loreggia, D., Makarov, V. V., Marseille, M. G., Martayan, C., Martinez-Rubi, O., Massart, B., Meynadier, F., Mignot, S., Munari, U., Nguyen, A. T., Nordlander, T., Ocvirk, P., O’Flaherty, K. S., Olias Sanz, A., Ortiz, P., Osorio, J., Oszkiewicz, D., Ouzounis, A., Palmer, M., Park, P., Pasquato, E., Peltzer, C., Peralta, J., Péturaud, F., Pieniluoma, T., Pigozzi, E., Poels, J., Prat, G., Prod'homme, T., Raison, F., Rebordao, J. M., Risquez, D., Rocca-Volmerange, B., Rosen, S., Ruiz-Fuertes, M. I., Russo, F., Sembay, S., Serraller Vizcaino, I., Short, A., Siebert, A., Silva, H., Sinachopoulos, D., Slezak, E., Soffel, M., Sosnowska, D., Straižys, V., ter Linden, M., Terrell, D., Theil, S., Tiede, C., Troisi, L., Tsalmantza, P., Tur, D., Vaccari, M., Vachier, F., Valles, P., Van Hamme, W., Veltz, L., Virtanen, J., Wallut, J. M., Wichmann, R., Wilkinson, M. I., Ziaeepour, H., and Zschocke, S., "The Gaia mission," Astronomy Er Astrophysics 595, A1 (Nov. 2016).

[26] Gaia Collaboration, Brown, A. G. A., Vallenari, A., Prusti, T., de Bruijne, J. H. J., Babusiaux, C., Biermann, M., Creevey, O. L., Evans, D. W., Eyer, L., Hutton, A., Jansen, F., Jordi, C., Klioner, S. A., Lammers, U., Lindegren, L., Luri, X., Mignard, F., Panem, C., Pourbaix, D., Randich, S., Sartoretti, P., Soubiran, C., Walton, N. A., Arenou, F., Bailer-Jones, C. A. L., Bastian, U., Cropper, M., Drimmel, R., Katz, D., Lattanzi, M. G., van Leeuwen, F., Bakker, J., Cacciari, C., Castañeda, J., De Angeli, F., Ducourant, C., Fabricius, C., Fouesneau, M., Frémat, Y., Guerra, R., Guerrier, A., Guiraud, J., Jean-Antoine Piccolo, A., Masana, E., Messineo, R., Mowlavi, N., Nicolas, C., Nienartowicz, K., Pailler, F., Panuzzo, P., Riclet, F., Roux, W., Seabroke, G. M., Sordo, R., Tanga, P., Thévenin, F., Gracia-Abril, G., Portell, J., Teyssier, D., Altmann, M., Andrae, R., Bellas-Velidis, I., Benson, K., Berthier, J., Blomme, R., Brugaletta, E., Burgess, P. W., Busso, G., Carry, B., Cellino, A., Cheek, N., Clementini, G., Damerdji, Y., Davidson, M., Delchambre, L., Dell'Oro, A., Fernández-Hernández, J., Galluccio, L., García-Lario, P., Garcia-Reinaldos, M., GonzálezNúñez, J., Gosset, E., Haigron, R., Halbwachs, J. L., Hambly, N. C., Harrison, D. L., Hatzidimitriou, D., Heiter, U., Hernández, J., Hestroffer, D., Hodgkin, S. T., Holl, B., Janßen, K., Jevardat de Fombelle, G., Jordan, S., Krone-Martins, A., Lanzafame, A. C., Löffler, W., Lorca, A., Manteiga, M., Marchal, O., Marrese, P. M., Moitinho, A., Mora, A., Muinonen, K., Osborne, P., Pancino, E., Pauwels, T., Petit, J. M., Recio-Blanco, A., Richards, P. J., Riello, M., Rimoldini, L., Robin, A. C., Roegiers, T., Rybizki, J., Sarro, L. M., Siopis, C., Smith, M., Sozzetti, A., Ulla, A., Utrilla, E., van Leeuwen, M., van Reeven, W., Abbas, U., Abreu Aramburu, A., Accart, S., Aerts, C., Aguado, J. J., Ajaj, M., Altavilla, G., Álvarez, M. A., Álvarez Cid-Fuentes, J., Alves, J., Anderson, R. I., Anglada Varela, E., Antoja, T., Audard, M., Baines, D., Baker, S. G., Balaguer-Núñez, L., Balbinot, E., Balog, Z., Barache, C., Barbato, D., Barros, M., Barstow, M. A., Bartolomé, S., Bassilana, J. L., Bauchet, N., Baudesson-Stella, A., Becciani, U., Bellazzini, M., Bernet, M., Bertone, S., Bianchi, L., Blanco-Cuaresma, S., Boch, T., Bombrun, A., Bossini, D., Bouquillon, S., Bragaglia, A., Bramante, L., Breedt, E., Bressan, A., Brouillet, N., Bucciarelli, B., Burlacu, A., Busonero, D., Butkevich, A. G., Buzzi, R., Caffau, E., Cancelliere, R., Cánovas, H., Cantat-Gaudin, T., Carballo, R., Carlucci, T., Carnerero, M. I., Carrasco, J. M., Casamiquela, L., Castellani, M., Castro-Ginard, A., Castro Sampol, P., Chaoul, L., Charlot, P., Chemin, L., Chiavassa, A., Cioni, M. R. L., Comoretto, G., Cooper, W. J., Cornez, T., Cowell, S., Crifo, F., Crosta, M., Crowley, C., Dafonte, C., Dapergolas, A., David, M., David, P., de Laverny, P., De Luise, F., De March, R., De Ridder, J., de Souza, R., de Teodoro, P., de Torres, A., del Peloso, E. F., del Pozo, E., Delbo, M., Delgado, A., Delgado, H. E., Delisle, J. B., Di Matteo, P., Diakite, S., Diener, C., Distefano, E., Dolding, C., Eappachen, D., Edvardsson, B., Enke, H., Esquej, P., Fabre, C., Fabrizio, M., Faigler, S., Fedorets, G., Fernique, P., Fienga, A., Figueras, F., Fouron, C., Fragkoudi, F., Fraile, E., Franke, F., Gai, M., Garabato, D., Garcia-Gutierrez, A., García-Torres, M., Garofalo, A., Gavras, P., Gerlach, E., Geyer, R., Giacobbe, P., Gilmore, G., Girona, S., Giuffrida, G., Gomel, R., Gomez, A., Gonzalez-Santamaria, I., González-Vidal, J. J., Granvik, M., Gutiérrez-Sánchez, R., Guy, L. P., Hauser, M., Haywood, M., Helmi, A., Hidalgo, S. L., Hilger, T., Hładczuk, N., Hobbs, D., Holland, G., Huckle, H. E., Jasniewicz, G., Jonker, P. G., Juaristi Campillo, J., Julbe, F., Karbevska, L., 
Kervella, P., Khanna, S., Kochoska, A., Kontizas, M., Kordopatis, G., Korn, A. J., Kostrzewa-Rutkowska, Z., Kruszyńska, K., Lambert, S., Lanza, A. F., Lasne, Y., Le Campion, J. F., Le Fustec, Y., Lebreton, Y., Lebzelter, T., Leccia, S., Leclerc, N., Lecoeur-Taibi, I., Liao, S., Licata, E., Lindstrøm, E. P., Lister, T. A., Livanou, E., Lobel, A., Madrero Pardo, P., Managau, S., Mann, R. G., Marchant, J. M., Marconi, M., Marcos Santos, M. M. S., Marinoni, S., Marocco, F., Marshall, D. J., Martin Polo, L., Martín-Fleitas, J. M., Masip, A., Massari, D., Mastrobuono-Battisti, A., Mazeh, T., McMillan, P. J., Messina, S., Michalik, D., Millar, N. R., Mints, A., Molina, D., Molinaro, R., Molnár, L., Montegriffo, P., Mor, R., Morbidelli, R., Morel, T., Morris, D., Mulone, A. F., Munoz, D., Muraveva, T., Murphy, C. P., Musella, I., Noval, L., Ordénovic, C., Orrù, G., Osinde, J., Pagani, C., Pagano, I., Palaversa, L., Palicio, P. A., Panahi, A., Pawlak, M., Peñalosa Esteller, X., Penttilä, A., Piersimoni, A. M., Pineau, F. X., Plachy, E., Plum, G., Poggio, E., Poretti, E., Poujoulet, E., Prša, A., Pulone, L., Racero, E., Ragaini, S., Rainer, M., Raiteri, C. M., Rambaux, N., Ramos, P., Ramos-Lerate, M., Re Fiorentin, P., Regibo, S., Reylé, C., Ripepi, V., Riva, A., Rixon, G., Robichon, N., Robin, C., Roelens, M., Rohrbasser, L., Romero-Gómez, M., Rowell, N., Royer, F., Rybicki, K. A., Sadowski, G., Sagristà Sellés, A., Sahlmann, J., Salgado, J., Salguero, E., Samaras, N., Sanchez Gimenez, V., Sanna, N., Santoveña, R., Sarasso, M., Schultheis, M., Sciacca, E., Segol, M., Segovia, J. C., Ségransan, D., Semeux, D., Shahaf, S., Siddiqui, H. I., Siebert, A., Siltala, L., Slezak, E., Smart, R. L., Solano, E., Solitro, F., Souami, D., Souchay, J., Spagna, A., Spoto, F., Steele, I. A., Steidelmüller, H., Stephenson, C. A., Süveges, M., Szabados, L., Szegedi-Elek, E., Taris, F., Tauran, G., Taylor, M. B., Teixeira, R., Thuillot, W., Tonello, N., Torra, F., Torra, J., Turon, C., Unger, N., Vaillant, M., van Dillen, E., Vanel, O., Vecchiato, A., Viala, Y., Vicente, D., Voutsinas, S., Weiler, M., Wevers, T., Wyrzykowski, Ł., Yoldas, A., Yvard, P., Zhao, H., Zorec, J., Zucker, S., Zurbach, C., and Zwitter, T., "Gaia Early Data Release 3. Summary of the contents and survey properties," Astronomy 83 Astrophysics 649, A1 (May 2021).

[27] Hashimoto, J., Tamura, M., Muto, T., Kudo, T., Fukagawa, M., Fukue, T., Goto, M., Grady, C. A., Henning, T., Hodapp, K., Honda, M., Inutsuka, S., Kokubo, E., Knapp, G., McElwain, M. W., Momose, M., Ohashi, N., Okamoto, Y. K., Takami, M., Turner, E. L., Wisniewski, J., Janson, M., Abe, L., Brandner, W., Carson, J., Egner, S., Feldt, M., Golota, T., Guyon, O., Hayano, Y., Hayashi, M., Hayashi, S., Ishii, M., Kandori, R., Kusakabe, N., Matsuo, T., Mayama, S., Miyama, S., Morino, J. I., Moro-Martin, A., Nishimura, T., Pyo, T. S., Suto, H., Suzuki, R., Takato, N., Terada, H., Thalmann, C., Tomono, D., Watanabe, M., Yamada, T., Takami, H., and Usuda, T., "Direct Imaging of Fine Structures in Giant Planet-forming Regions of the Protoplanetary Disk Around AB Aurigae," Astrophysical Journal Letters 729, L17 (Mar. 2011).

[28] Boccaletti, A., Di Folco, E., Pantin, E., Dutrey, A., Guilloteau, S., Tang, Y. W., Piétu, V., Habart, E., Milli, J., Beck, T. L., and Maire, A. L., "Possible evidence of ongoing planet formation in AB Aurigae. A showcase of the SPHERE/ALMA synergy," Astronomy 83 Astrophysics 637, L5 (May 2020).

[29] Grady, C. A., Woodgate, B., Bruhweiler, F. C., Boggess, A., Plait, P., Lindler, D. J., Clampin, M., and Kalas, P., "Hubble Space Telescope Space Telescope Imaging Spectrograph Coronagraphic Imaging of the Herbig AE Star AB Aurigae," Astrophysical Journal Letters 523, L151-L154 (Oct. 1999).

[30] Fukagawa, M., Hayashi, M., Tamura, M., Itoh, Y., Hayashi, S. S., Oasa, Y., Takeuchi, T., Morino, J.-i., Murakawa, K., Oya, S., Yamashita, T., Suto, H., Mayama, S., Naoi, T., Ishii, M., Pyo, T.-S., Nishikawa, T., Takato, N., Usuda, T., Ando, H., Iye, M., Miyama, S. M., and Kaifu, N., "Spiral Structure in the Circumstellar Disk around AB Aurigae," Astrophysical Journal Letters 605, L53-L56 (Apr. 2004).

[31] Hoff, W., Henning, T., and Pfau, W., "The nature of isolated T Tauri stars," Astronomy 83 Astrophysics 336, 242-250 (Aug. 1998).

[32] Barrado Y Navascués, D., "On the age of the TW Hydrae association and 2M1207334-393254," Astronomy \& Astrophysics 459, 511-518 (Nov. 2006).

[33] Vacca, W. D. and Sandell, G., "Near-infrared Spectroscopy of TW Hya: A Revised Spectral Type and Comparison with Magnetospheric Accretion Models," Astrophysical Journal 732, 8 (May 2011).

[34] van Boekel, R., Henning, T., Menu, J., de Boer, J., Langlois, M., Müller, A., Avenhaus, H., Boccaletti, A., Schmid, H. M., Thalmann, C., Benisty, M., Dominik, C., Ginski, C., Girard, J. H., Gisler, D., Lobo Gomes, A., Menard, F., Min, M., Pavlov, A., Pohl, A., Quanz, S. P., Rabou, P., Roelfsema, R., Sauvage, J. F., Teague, R., Wildi, F., and Zurlo, A., "Three Radial Gaps in the Disk of TW Hydrae Imaged with SPHERE," Astrophysical Journal 837, 132 (Mar. 2017). 
[35] Akiyama, E., Muto, T., Kusakabe, N., Kataoka, A., Hashimoto, J., Tsukagoshi, T., Kwon, J., Kudo, T., Kandori, R., Grady, C. A., Takami, M., Janson, M., Kuzuhara, M., Henning, T., Sitko, M. L., Carson, J. C., Mayama, S., Currie, T., Thalmann, C., Wisniewski, J., Momose, M., Ohashi, N., Abe, L., Brandner, W., Brandt, T. D., Egner, S., Feldt, M., Goto, M., Guyon, O., Hayano, Y., Hayashi, M., Hayashi, S., Hodapp, K. W., Ishi, M., Iye, M., Knapp, G. R., Matsuo, T., Mcelwain, M. W., Miyama, S., Morino, J. I., Moro-Martin, A., Nishimura, T., Pyo, T. S., Serabyn, G., Suenaga, T., Suto, H., Suzuki, R., Takahashi, Y. H., Takato, N., Terada, H., Tomono, D., Turner, E. L., Watanabe, M., Yamada, T., Takami, H., Usuda, T., and Tamura, M., "Discovery of a Disk Gap Candidate at 20 AU in TW Hydrae," Astrophysical Journal Letters 802, L17 (Apr. 2015).

[36] Brandt, T. D., Rizzo, M., Groff, T., Chilcote, J., Greco, J. P., Kasdin, N. J., Limbach, M. A., Galvin, M., Loomis, C., Knapp, G., McElwain, M. W., Jovanovic, N., Currie, T., Mede, K., Tamura, M., Takato, N., and Hayashi, M., "Data reduction pipeline for the CHARIS integral-field spectrograph I: detector readout calibration and data cube extraction," Journal of Astronomical Telescopes, Instruments, and Systems 3, 048002 (Oct. 2017).

[37] Currie, T., Brandt, T. D., Uyama, T., Nielsen, E. L., Blunt, S., Guyon, O., Tamura, M., Marois, C., Mede, K., Kuzuhara, M., Groff, T. D., Jovanovic, N., Kasdin, N. J., Lozi, J., Hodapp, K., Chilcote, J., Carson, J., Martinache, F., Goebel, S., Grady, C., McElwain, M., Akiyama, E., Asensio-Torres, R., Hayashi, M., Janson, M., Knapp, G. R., Kwon, J., Nishikawa, J., Oh, D., Schlieder, J., Serabyn, E., Sitko, M., and Skaf, N., "SCExAO/CHARIS Near-infrared Direct Imaging, Spectroscopy, and Forward-Modeling of $\kappa$ And b: A Likely Young, Low-gravity Superjovian Companion," Astronomical Journal 156, 291 (Dec 2018).

[38] Ren, B., Dong, R., van Holstein, R. G., Ruffio, J.-B., Calvin, B. A., Girard, J. H., Benisty, M., Boccaletti, A., Esposito, T. M., Choquet, É., Mawet, D., Pueyo, L., Stolker, T., Chiang, E., Boer, J., Debes, J. H., Garufi, A., Grady, C. A., Hines, D. C., Maire, A.-L., Ménard, F., Millar-Blanchaer, M. A., Perrin, M. D., Poteet, C. A., and Schneider, G., "Dynamical Evidence of a Spiral Arm-driving Planet in the MWC 758 Protoplanetary Disk," Astrophysical Journal Letters 898, L38 (Aug. 2020).

[39] Hughes, A. M., Duchêne, G., and Matthews, B. C., "Debris Disks: Structure, Composition, and Variability," Annual Reviews of Astronomy \&3 Astrophysics 56, 541-591 (Sept. 2018). 